\title{
Importance of sulfur and its roles in Plants physiology: A Review
}

\author{
${ }^{1}$ Abou Seeda M.A., ${ }^{2}$ Abou El-Nour E.A.A., ${ }^{1}$ Yassen A.A., ${ }^{3}$ Gad Mervat M. and ${ }^{1}$ Sahar M. \\ Zaghloul
}

${ }^{1}$ Plant Nutrition Dept., National Research Centre, 33 El Buhouth St., 12622 Dokki, Giza, Egypt.

${ }^{2}$ Fertilization Tech. Dept., National Research Centre, 33 El Buhouth St., 12622 Dokki, Giza, Egypt.

${ }^{3}$ Timber Trees Dept. Horticulture Research Institute Agricultural Research Centre, Giza, Egypt.

Received: 05 Feb. 2020 / Accepted 08 April 2020 / Publication date: 20 April 2020

\begin{abstract}
Sulfur is one of the most versatile elements in life. It functions in fundamental processes such as electron transport, structure, and regulation. In plants, additional roles have developed with respect to photosynthetic oxygen production. Sulfate uptake, reductive assimilation, and integration into cysteine and methionine are the central processes that direct oxidized and reduced forms of organically bound sulfur into its various functions. Sulfite reductase (SIR) is a key enzyme in higher plants in the assimilatory sulfate reduction pathway. The reduction of SIR activity caused chlorotic and necrotic phenotypes in tobacco leaves, but with varying phenotype strength even among clones and increasing from young to old leaves. The levels of downstream metabolites were reduced, such as cysteine, glutathione (GSH) and methionine. This metabolic signature resembles a sulfate deprivation phenotype as corroborated by the fact that $\mathrm{O}$-acetylserine (OAS) accumulated. In addition, chlorophyll contents, photosynthetic electron transport, and the contents of carbohydrates such as starch, sucrose, fructose, and glucose were reduced. Amino acid compositions were altered in a complex manner due to the reduction of contents of cysteine, and to some extent methionine. Interestingly, sulfide levels remained constant indicating that sulfide homeostasis is crucial for plant performance and survival
\end{abstract}

Keywords: Importance of sulfur, Sulfur transporter, (Cys) Cysteine, (Mys) Methionine. (Gys) Glutathione

\section{Introduction}

Recent studies have demonstrated that sulfur is importantlfor the proper growth, metabolic activities, and development of plants. Sulfur is one of the most essential macronutrients required by the plants, as it is an important constituent of amino acids such as cysteine and methionine and in many metabolites Leustek and. Saito (1999).Cysteine, as the first organic reduced sulfur compound, and methionine and its derivatives contribute to life not only as building blocks in proteins and their activity, but also as precursors for the synthesis of glutathione (GSH), cofactors (like Fe-S clusters, heme, siroheme, molybdenum centres, and lipoic acid) Fig.(1).

Essential vitamins (biotin and thiamine), sulfur esters (coenzyme A), and sulfur derivatives. Sulfur is also an important constituent of several coenzymes, thioredoxins, and sulpholipids. Sulfur is also an important constituent of some compounds with may be involved in defense mechanisms against herbivores, pests, and pathogens or constituents to the special taste and odour of food plants Bennett and Walls grove (1994). Glutathione is a major thiol containing metabolite often present in millimolar concentrations and associated with the defense system. Sulfur is available primarily in the form of anionic sulfate $\left(\mathrm{SO}_{4}{ }^{-2}\right)$ to plants which is transported through roots and then distributed via xylem to stem and other parts of plants Leustek and. Saito (1999).Visible symptoms of sulfur deficiency such as chlorosis appear first in young leaves while older leaves remain green, suggesting that sulfur is immobile in older leaves. Insoluble sulfur deficient conditions Sunarpi and Anderson (1996), Sulfur deficiency also affects $\mathrm{CO}_{2}$ assimilation rates and rubisco enzyme activities and protein abundance Gilbert et al. (1997). Reactive oxygen species (ROS) are generally produced during cellular metabolism and cells are always ready to cope up with this condition through their antioxidative machinery and scavenging enzymes. Nutrient deficiencies are also responsible for ROS production Mittler (2006) Jaspers and. Kangasj (2010) Chloroplast is an important source of producing ROS like superoxide $\left(\mathrm{O}^{-}\right.$ $\left.{ }^{2}\right)$ and hydrogen peroxide $\left(\mathrm{H}_{2} \mathrm{O}_{2}\right)$ during reduced rate of photosynthetic carbon fixation Takahashi and

Corresponding Author: Abou Seeda M.A., Plant Nutrition Dept., National Research Centre, 33 El Buhouth St., 12622 Dokki, Giza, Egypt. E-mail: mabouseeda@gmail.com 
Murata (2008).Chloroplast can also produce ROS like singlet oxygen (1O2) through excited chlorophyll molecule Triantaphylid'es and Havaux, (2009).Mitochondria and peroxisomes are also responsible for ROS production. In addition to these, ROS like hydroxyl radical $\left(\mathrm{OH}^{-}\right)$can be formed from $\mathrm{H}_{2} \mathrm{O}_{2}$ and $\mathrm{O}^{-2}$ through Haber-Weiss reaction Miler et al. (2007) Fig. (2).

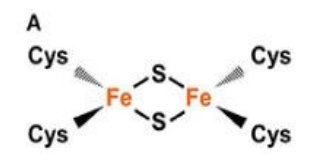

D

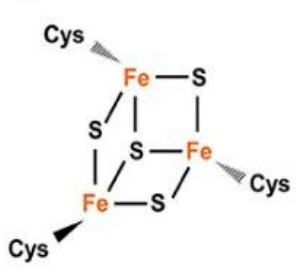

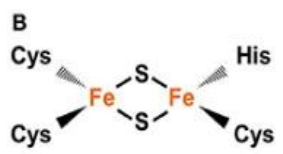

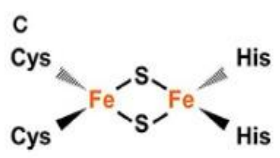

E

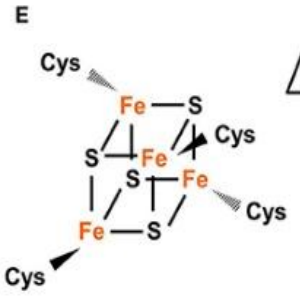

Fig. 1: Common types of Fe-S clusters found in the plastid.

(a) Classic $2 \mathrm{Fe}-2 \mathrm{~S}$ coordinated by four Cys residues, as in the plant-type $\mathrm{Fd}$ (ferredoxin).

(b) NEET-type $2 \mathrm{Fe}-2 \mathrm{~S}$ coordinated by three Cys and one His residues, as in NEET.

(c) Rieske-type $2 \mathrm{Fe}-2 \mathrm{~S}$ coordinated by two Cys and two His residues, as in PetC

(Photosynthetic electron transfer C).

(d) $3 \mathrm{Fe}-4 \mathrm{~S}$ coordinated by three Cys residues as in Fd-GOGATs (ferredoxin-dependent

(Gln oxoglutarate aminotransferases).

(e) $4 \mathrm{Fe}-4 \mathrm{~S}$ coordinated by four Cys residues, as in PsaA, PsaB, and PsaC

(Photosystem I proteins A-C).

(f) $4 \mathrm{Fe}-4 \mathrm{~S}$ coordinated by four Cys residues with a thiolate ligand serving also for siroheme, as in NiR (nitrite reductase) and $\mathrm{SiR}$ (sulfite reductase). In Fe-S clusters, the oxidation state of $\mathrm{Fe}$ could be $\mathrm{Fe}+2$ or $\mathrm{Fe}+3$ while the oxidation state of $\mathrm{S}$ » $\mathrm{s}-2$

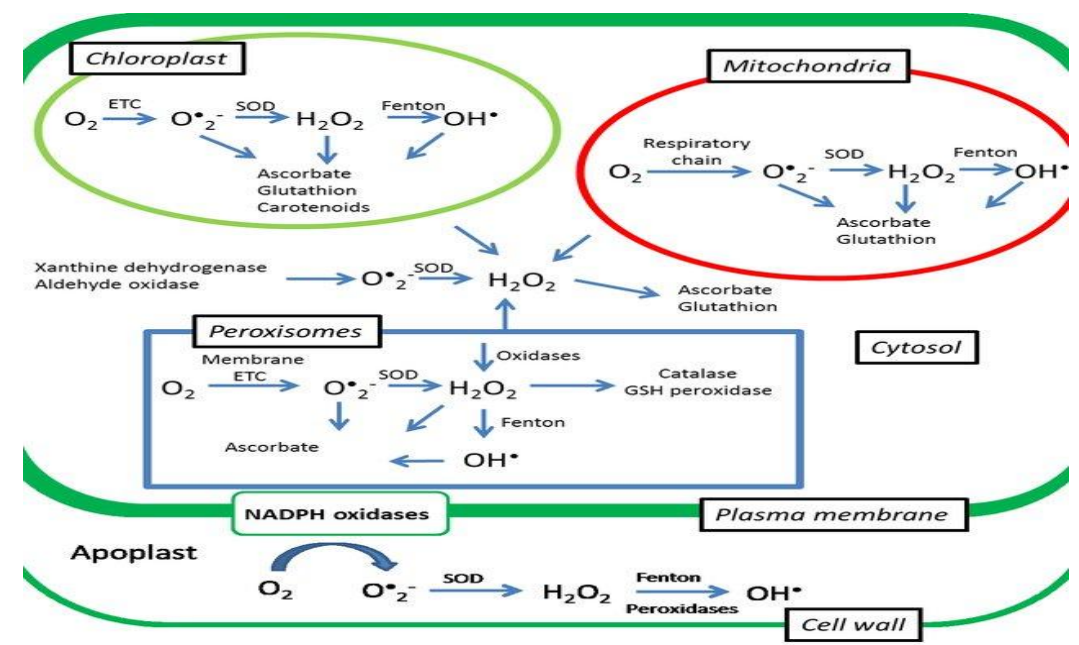

Fig. 2: Fenton (decomposition of hydrogen peroxide to highly reactive hydroxyl radical in presence of iron); ETC (electron transport chain)

\section{1- Sulfur is an essential element}

Improving crop productivity through the adoption of high-yielding varieties and multiple cropping systems, fertilizer use has become more and more important to increase crops yield and quality. Sulphur is an essential plant nutrient for crop production. For oil crop producers, Sulphur fertilizer is especially important because oil crops require more Sulphur than cereal grains. For example, the amount of 
Sulphur required to produce one ton of seed is about 3-4 kg Sulphur for cereals (range 1-6); $8 \mathrm{~kg}$ Sulphur for legume crops (range 5-13); and $12 \mathrm{~kg}$ Sulphur for oil crops (range 5-20). In general, oil crops require about the same amount of Sulphur as, or more than, phosphorus for high yield and product quality. In intensive crop rotations including oil crops, Sulphur uptake can be very high, especially when the crop residue is removed from the field along with the product. This leads to considerable Sulphur depletion in soil if the corresponding amount of Sulphur is not applied through fertilizer. Sulphur is increasingly being recognized as the fourth major plant nutrient after nitrogen, phosphorus and potassium. The importance of Sulphur in agriculture is being increasingly emphasized and its role in crop production is well

Recognized Jamal et al., (2005), (2006a); (2006b); (2006c); (2009); (2010); Scherer, (2009). Sulphur is best known for its role in the formation of amino acids methionine ( $21 \%$ Sulphur) and cysteine $(27 \%$ Sulphur); synthesis of proteins and chlorophyll; oil content of the seeds and nutritive quality of forages Tandon, (1986); Jamal et al., (2005), (2006a); (2009) Fig.(3).

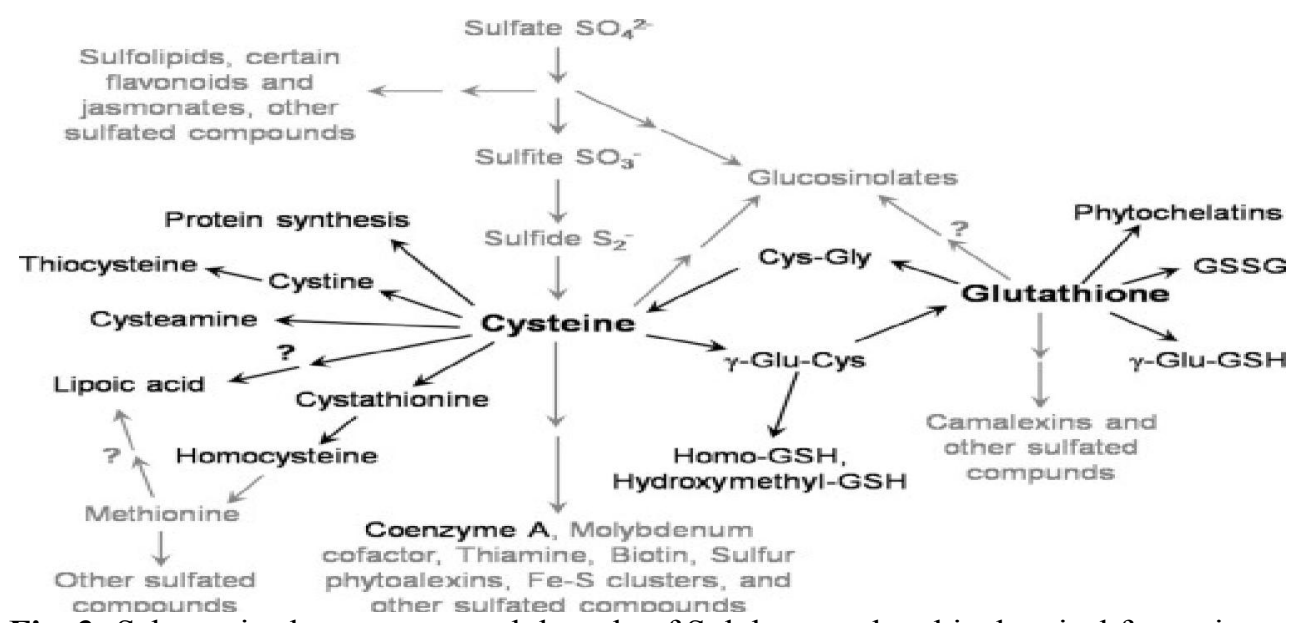

Fig. 3: Schematic chart represented the role of Sulphur on plant biochemical formation

Although Sulphur is one of the essential nutrients for plant growth with crop requirement similar to phosphorus, this element received little attention for many years, because fertilizers and atmospheric inputs supplied the soil with adequate amounts of Sulphur Fig. (4).
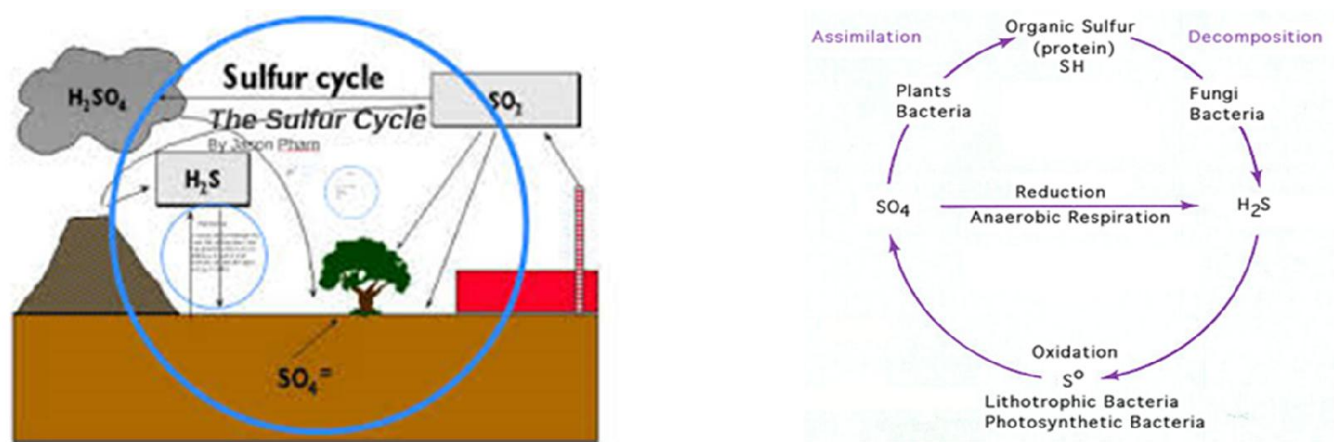

Fig. 4: Schematic diagram represented Sulfur Cycle

Now, areas of Sulphur deficiency are becoming widespread throughout the world due to the use of high-analysis low Sulphur fertilizers, low Sulphur returns with farmyard manure, high yielding varieties and intensive agriculture, declining use of Sulphur containing fungicides and reduced atmospheric input caused by stricter emission regulation. An insufficient Sulphur supply can affect yield and quality of the crops; caused by the Sulphur requirement for protein and enzyme synthesis as well it is a constituent of the amino acids, methionine and cysteine Fig.(5). 


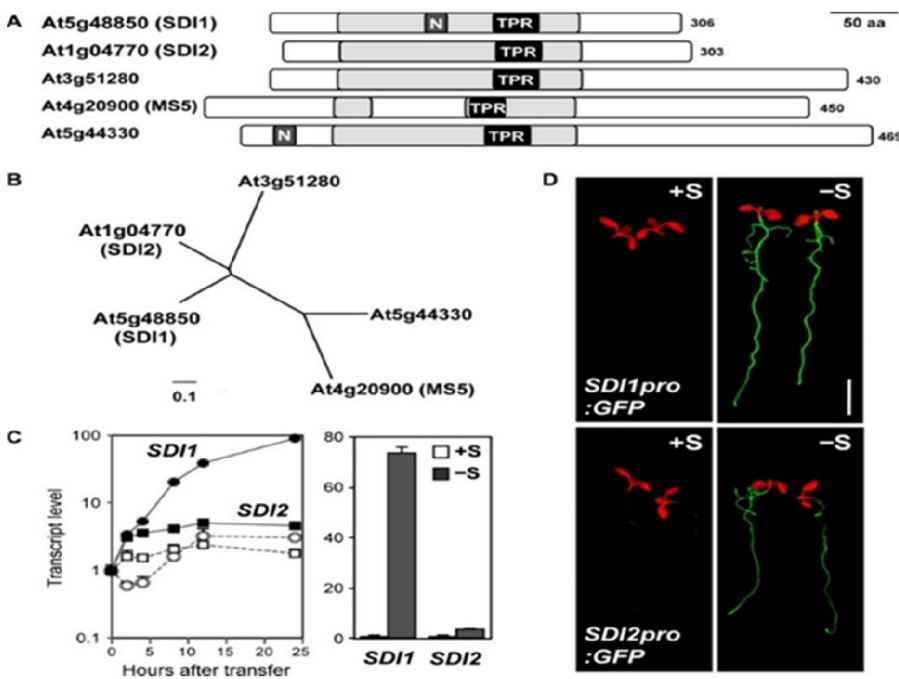

Fig. 5: Represents SDI1 and SDI2 are TPR proteins induced by sulfur deficiency in Arabidopsis. $(\boldsymbol{A})$ Schematic representation of the TPR domain that exists in Arabidopsis SDI proteins. TPR-like helical domains detected by InterPro scan [pale gray bar Lamb, et al. (1995), TPR motifs; black bar with TPR, TPR1, and PF00515] and nuclear localization signals (dark gray bar with N, NLS BP, and PS50079) detected by Motif Scan (64) were indicated. Numbers on the right represent amino acid length of each protein. Scale bar, 50 amino acids (AA). (B) Phylogenetic relationships of SDI family proteins in Arabidopsis. Protein sequences of SDI1 (At5g48850), SDI2 (At1g04770), At3g51280, MS5 (At4g20900), and MS5-like (At5g44330) were obtained from The Arabidopsis Information Resources (TAIR) (www.arabidopsis.org). They were aligned using a Clustal W program, and an unrooted phylogenetic tree was drawn by neighbor-joining methods using the Geneious software (Biomatters Ltd.). (C) Induction of SDI1 and SDI2 by sulfur deficiency. (Left) Transcript accumulation of SDI1 (circle) and SDI2 (square) in roots of WT transferred under $+\mathrm{S}$ to $+\mathrm{S}$ (open markers) or $+\mathrm{S}$ to $-\mathrm{S}$ (filled markers) conditions detected by previous microarray experiments (Maruyama-Nakashita et al. (2005). (Right) Transcript levels of SDI1 and SDI2 in roots of WT grown under $+\mathrm{S}$ (1500 mM sulfate, white bars) or -S (15 mM sulfate, gray bars) conditions detected by previous microarray experiments [means \pm SE of duplicates (MaruyamaNakashita et al. (2006). (D) Green fluorescent protein (GFP) accumulation in SDI1pro: GFP and SDI2pro: GFP plants grown under $+\mathrm{S}$ and $-\mathrm{S}$ conditions. GFP fluorescence was visualized under an image analyzer, as described in Materials and Methods. Scale bar, 5 mm. after Aarabi et al. (2016)

Sulfur toxicity is very rare and will most likely not occur even if excessive sulfuric acid is injected to neutralize water alkalinity. High sulfur levels in the growing medium can compete with and induce nitrogen deficiency as presented in Fig. (6). To overcome the problems associated with Sulphur deficiency a number of Sulphur-containing fertilizers as well as other Sulphur containing by-products from industrial processes are available.
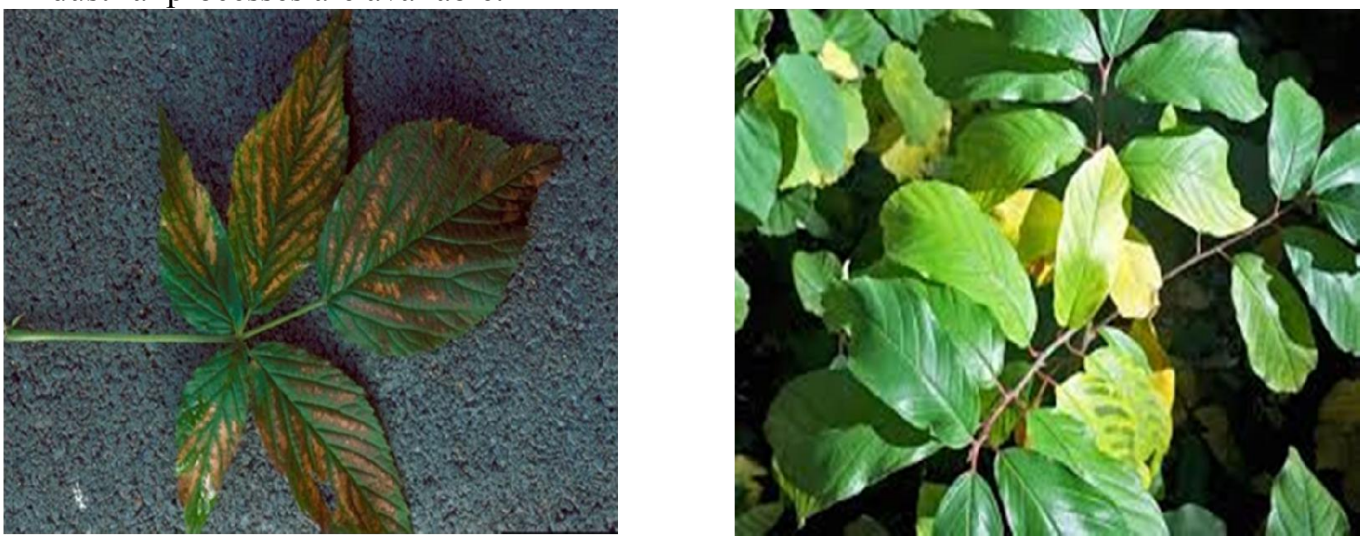

Fig. 6: Illustrates toxicity symptoms of Sulphur

Sulfur toxicity is very rare and will most likely not occur even if excessive sulfuric acid is injected to neutralize water alkalinity. High sulfur levels in the growing medium can compete with and induce nitrogen deficiency. To overcome the problems associated with Sulphur deficiency a number of 
Sulphur-containing fertilizers as well as other Sulphur containing by-products from industrial processes are available.

Sulfur is a crucial microelement for plant nutrition, required by the biosynthesis of sulfolipids, antioxidants, cofactors, secondary metabolites, and amino acids that are strictly or conditionally essential for human nutrition (cysteine and methionine) Hawkesford, and De Kok (2006), Stipanuk (2004). In addition, grain S content is essential to form protein disulfide bridges and dictates flourprocessing properties (such as dough extensibility) in bread, pasta or biscuit industry Zhao et al. (2011).

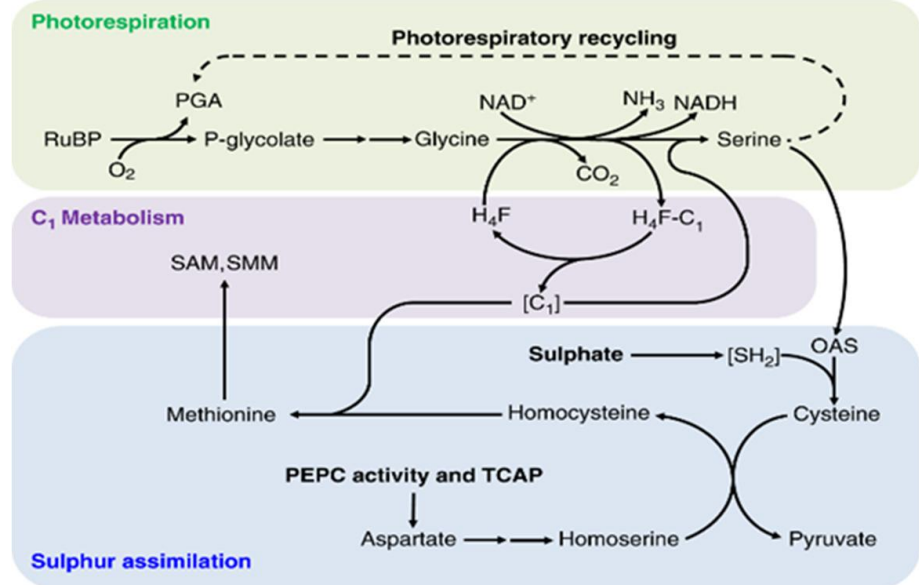

Fig. 7: Represents rationale of metabolic interactions between photorespiration and $\mathrm{S}$ assimilation in plant leaves. Schematic diagram shows photorespiration could be beneficial to sulfur assimilation. Photorespiration drives the production of serine that is used to synthesize cysteine de novo. In addition, photorespiration involves $\mathrm{C} 1$ metabolism from which methyl-tetrahydrofolate (H4F) can be used to synthesize methionine de novo. OAS Oacetylserine,PEPC phosphoenolpyruvate carboxylase, PGA 3-phosphoglycerate, RuBP ribulose 1,5-bisphosphate, SAM S-adenosyl methionine, SMM Smethylmethionine,TCAP tricarboxylic acid pathway ("Krebs cycle"). For simplicity, the SAM-SMM cycle that can reform methionine

It is often assumed that $\mathrm{S}$ assimilation positively correlates with photosynthesis, because sulfate incorporation is stimulated by sugars in plant roots Brunold, (2003) Fig. (7), and inhibited under nonphysiological conditions where $\mathrm{CO}_{2}$ is omitted from the atmosphere $\left(\mathrm{CO}_{2}\right.$-free air) Kopriva, (2002). Nevertheless, the relationship with photosynthesis has never been tested experimentally and thus it is presently uncertain as to whether Sulphur assimilation can be impacted by environmental conditions that affect photosynthetic metabolism. As mentioned above, solving the question of a possible correlation between photosynthesis and $\mathrm{S}$ metabolism in the long term is critically important because $\mathrm{CO}_{2}$ mole fraction in Earth's atmosphere inexorably increases and favours plant photosynthesis, and also current metabolic engineering aims to increase photosynthesis by suppressing photorespiration Kulick,(2013), South(2019). In the past 20 years, it has been found that plants cultivated in a $\mathrm{CO}_{2}$ enriched atmosphere contain less elemental Sulphur Jia, (2010), Ågren, and Weih,(2012) including in grains, thereby affecting flour quality Högy, and Fangmeier (2008) Högy,(2009). Accordingly, cultivation under $\mathrm{CO}_{2}$-enriched conditions has been found to alter the content in Sulphur containing antioxidant (glutathione) Badiani et al. (1993) Schulte, et al. (2002). Therefore, present atmospheric changes (probable $\mathrm{CO}_{2}$ doubling by 2100) seem to be detrimental to Sulphur metabolism. This problem may worsen in the near future because of limited available Sulphur in the environment, due to decreased pollution-driven $\mathrm{SO}_{2}$ emissions and lower utilization of Sulphur containing fertilizers (such as superphosphate). However, possible mechanisms explaining the lower Sulphur content when plants are subjected to high $\mathrm{CO}_{2}$ have not been studied.

Possible correlation between photosynthesis and Sulphur assimilation in the short term is also important since in the field, photosynthesis can vary considerably depending on environmental conditions, and thus plant Sulphur nutrition could be affected accordingly. Furthermore, Sulphur metabolism is involved in electron consumption and redox metabolism in illuminated leaves and thus can affect leaf photosynthetic capacity Chan, et al. (2013). In principle, changes in S metabolism when photosynthesis varies could stem from metabolic interactions with photorespiration. The key enzymatic activity of photo respiratory metabolism is the conversion of glycine to serine. In this biochemically 
complicated reaction, a glycine molecule is cleaved thereby liberating $\mathrm{CO}_{2}$ and ammonium $\mathrm{NH}_{4}$, and a one-carbon unit is fixed onto another glycine molecule to form serine. This reaction involves tetrahydrofolate $\left(\mathrm{H}_{4} \mathrm{~F}\right)$ as a cofactor to transfer the one carbon unit Douce et al. (2001). One-carbon (C1) metabolism is thus directly involved in photorespiration (due to H4F requirement) and this is visible in, e.g., mutants affected in formyl tetrahydrofolate deformylases (which regenerates $\mathrm{H}_{4} \mathrm{~F}$ ) that have growth defects and accumulate glycine to very high levels under photo respiratory conditions Collakova (2008). In addition, C1 metabolism is essential not only for photorespiration but also for many one-carbon requiring reactions of metabolism, including methionine synthesis 21 . The involvement of $\mathrm{N}$-containing compounds (ammonium, glycine, and serine) in photorespiration explains why photorespiration is intimately linked to enzyme activities of $\mathrm{N}$ assimilation and how it may stimulate nitrate reduction Bloom et al (2010), Rachmilevitch, et al (2004). There is no such direct relationship with sulfur but presumably, photorespiration may stimulate Sulphur assimilation because serine and one-carbon units are the building blocks used to synthesize S-containing amino acids methionine and cysteine.

\section{2-1 Physiological Functions of Sulfur}

Sulfur is of elemental importance for life due to its versatility and reactivity in different oxidation and reduction states. In phototrophic organisms in general, the redox properties of sulfur in proteins and in sulfur-containing metabolites are important as mediators between the reductive assimilation processes of photosynthesis and reactive oxygen species that arise as byproducts of electron transport chains. Reduced sulfur compounds have been assumed as early energy donors and, together with iron, may have contributed to early electron transport processes in proto-life Martin et al. (2003). The reductive conditions before the invention of oxygenic photosynthesis probably allowed the evolution of processes based on the redox properties of sulfur Fig. (8).
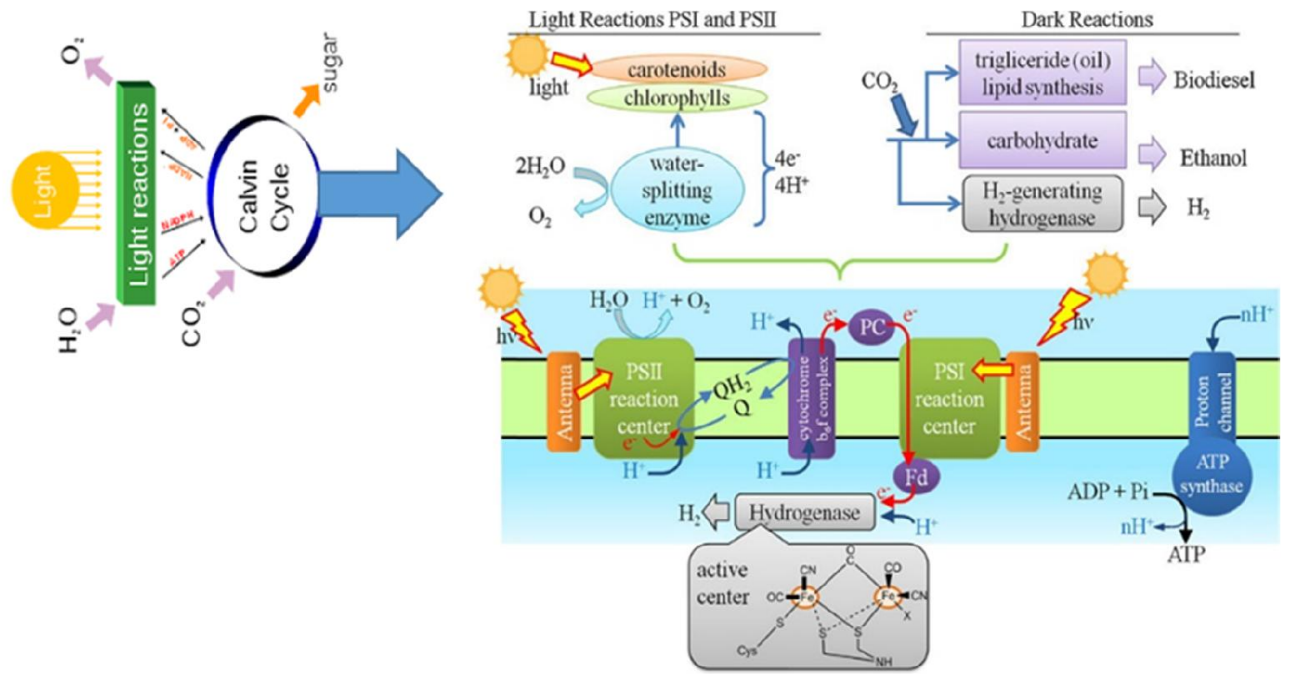

Fig. 8: Schematic diagram of light and dark reaction

Schematic drawing shows light energy conversion processes carried out in photosynthetic membranes of oxygenic phototrophs, such as plants. Plant photosynthesis is a complicated series of redox reactions, using light energy from the sun and electrons donated from the splitting of water, that produce bioenergy (ATP-adenosine triphosphate) and reducing equivalents (NADPH-the reduced form of nicotine adenine dinucleotide phosphoric acid), which are subsequently consumed in the reduction of carbon dioxide to produce a fuel (carbohydrates). During these processes, molecular oxygen is produced as a final by-product. In other words, photosynthesis produces all the food that is eaten and is the origin of all food chains. It also produces all the oxygen that we breathe. Therefore, photosynthesis is ultimately the source of all life inhabiting the Earth. Light harvesting pigment-protein complexes (also called photosynthetic antenna proteins) absorb light energy from the sun to initiate the abovementioned photosynthetic reactions. 
Thus, harvested solar energy is transferred in the form of excitation energy to one of two reaction center (RC) complexes, either Photosystem II (PSII) or Photosystem I (PSI). In these RC complexes, an electric charge separation reaction takes place through the subsequent redox reactions and electromotive force is accumulated. The synthesis of ATP and NADPH are dependent upon this electromotive force. PSII holds an oxidation catalyst and PSI holds the reduction catalyst. PSII has the Quinone type RC that is similar to purple photosynthetic bacteria and PSI contains the iron-sulfur cluster type RC that is similar to green sulfur bacteria. A remarkable feature of PSII is that the oxidative splitting of water molecules fills the positive 'hole', produced on the P $680+\mathrm{Chl}$ dimer because of the loss of an electron during electric charge separation. This reaction is unique in biology and is performed by a biocatalyst.

These sulfur-based reduction systems remained when the atmospheric environment became oxidative because of the effective development of photosynthesis in bacteria and later in algae and plants. It was suggested that this transition promoted the evolution of oxygen dependent pathways as basis of subsequently complex organismal development Falkowski (2006). In addition to iron-sulfur clusters, the catalytic abilities of reduced sulfur are documented in the cofactors and ligands coenzyme A, biotin, thiamin, lipoic acid, and others. They are of fundamental importance to all cells since the very origin of life and have essential functions as vitamins in mammals. In proteins, the structural role of disulfide bridges is well established and may be renewed in this chapter by the discovery of two functionally important disulfide bridges in plant g-glutamylcysteine ligase (GSH1), the first enzyme of GSH biosynthesis Jez et al. (2004); Hothorn et al. (2006); Gromes et al. (2008). Regulatory functions of disulfide bridges in plants are prominent in enzymes of the Calvin Cycle but are found in increasing numbers in control switches such as NPR1 in pathogen defence Tada et al. (2008) and transcription factors Ndamukong et al. (2007). Monothiol-and dithiol mediated transfer processes are essential in these highly specific and directed processes that include GSH, NO, thioredoxins and glutaredoxins Meyer and Hell (2005); Meyer et al. (2008). These primary cellular functions are, in plants, complemented by the so-called secondary sulfur compounds. Many of them have roles in plant defence processes against hytopathogenic microbes and pests, such as the phytoalexin camalexin, sulfur-rich peptides of the thionin and defensin groups, and glucosinolates in the Brassicaceae family Rausch and Wachter (2005). Lack of these compounds reduces the defence capabilities, while their formation upon attack goes along with an activation of primary sulfur metabolism. Since the synthesis of these defence compounds seems to partially depend on optimal sulfate supply, the term sulfur enhanced defence" has been coined Kruse et al. (2007). The many aspects of sulfur function in phototrophic organisms have recently been comprehensively reviewed Dahl et al. (2008).

\section{2-2 Symptoms of Sulfur Deficiency}

The numerous functions of sulfur in plants give rise to a characteristic long-term deficiency phenotype: chlorosis of interveinal sections of young leaves Photo. (1).

The lack of reduced sulfur for iron-sulfur clusters in photosynthesis is presumably one reason that gives rise to chlorophyll oxidation; degradation of photosynthetic proteins to regain reduced sulfur is another Ferreira and Teixeira (1992); Gilbert et al. (1997). In contrast to nitrate metabolism, where mature leaves under nitrogen starvation turn chlorotic because they export nitrate to young sink leaves for growth, the young leaves react first and strongest to prolonged sulfate deficiency. The reason for this difference is seen in a slow release of sulfate stored in the vacuoles of mature leaves Bell et al. (1994). In comparison, the mobility of nitrate between vacuole and cytosol upon demand is much higher Miller et al. (2009). The activation rate of sulfate seems to be connected to nitrogen availability and growth rate Blake-Kalff et al. (1998); Dubousset et al. (2009). This physiological feature gives rise to problems with early diagnosis of sulfur deficiency in agriculture. Determination of free sulfate in leaves is not indicative of the sulfate supply of a crop. Leaf sulfate contents may still be high, but are of only limited use, while the plant already suffers from sulfate deficiency in the soil solution Blake-Kalff et al. (1998). 

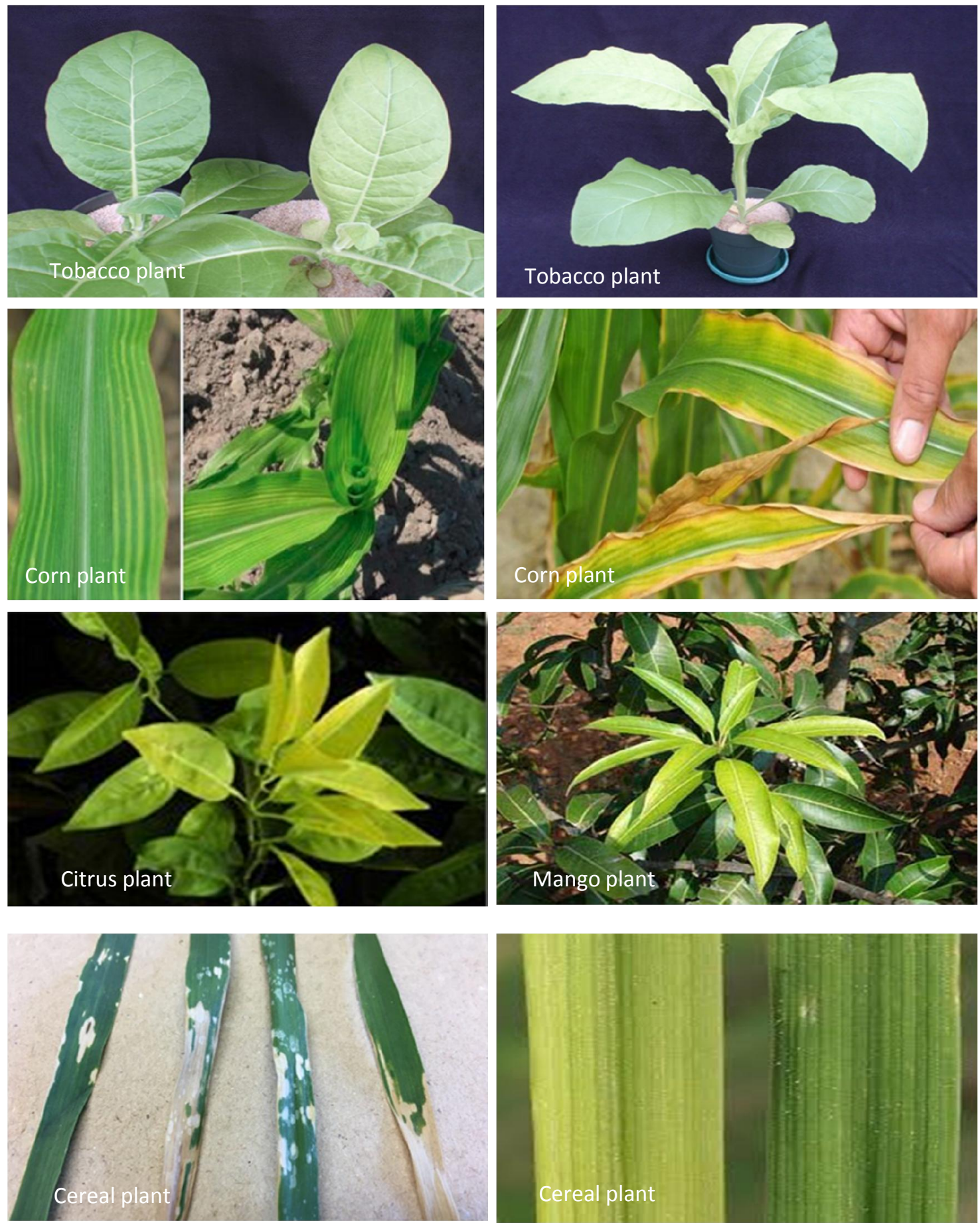

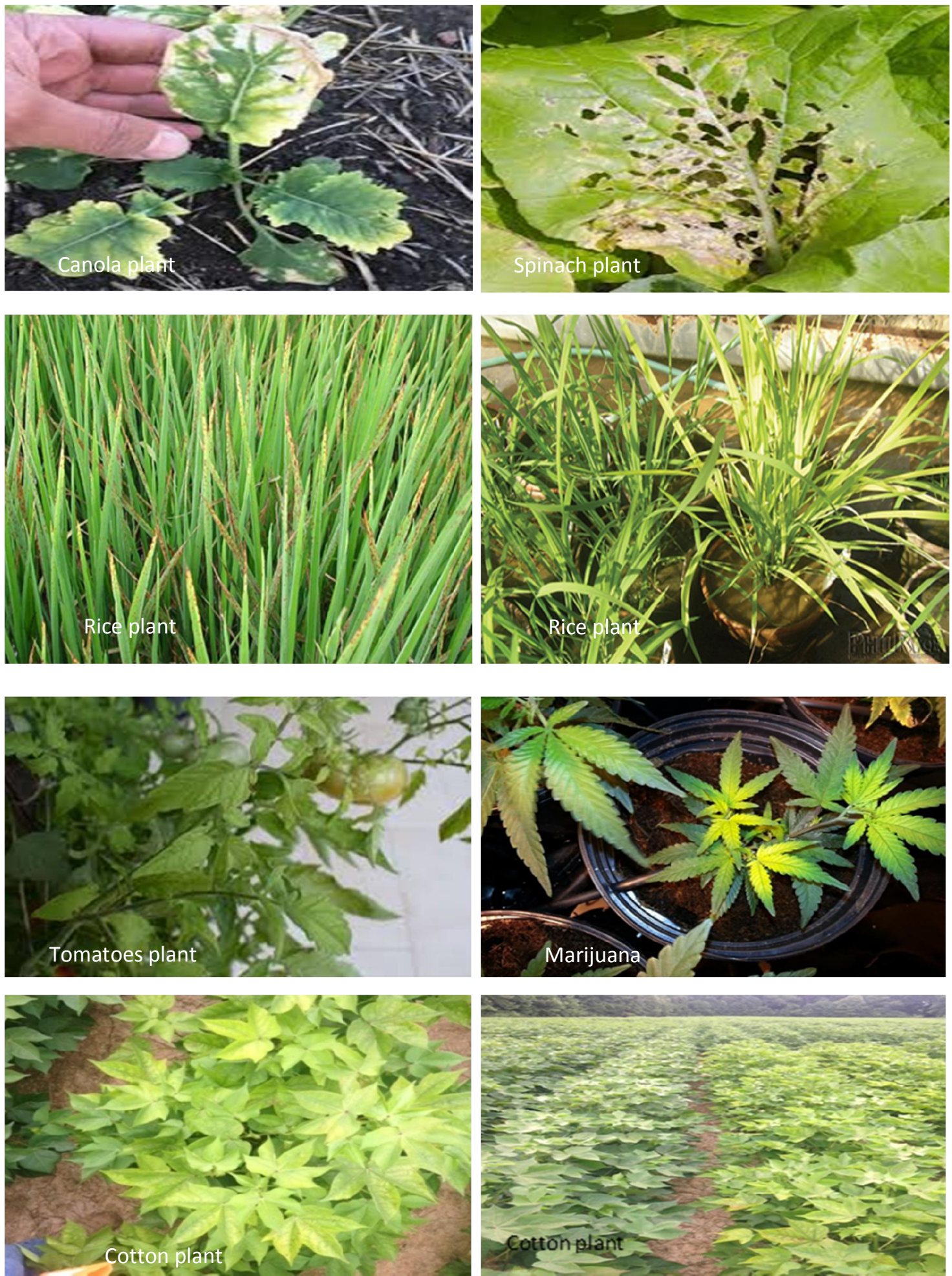

Photo.1: Illustrates some deficiency symptoms of elemental sulphur

Marcell Nauman (2018) reported that in the Sulfite reductase (SIR) Co-suppression lines E2, P2, and P3 metabolites of the primary sulfate metabolism were determined in order to investigate the effect of the introduced bottleneck in the primary sulfur assimilation pathway. Free ionic sulfate levels increased significantly in most samples in comparison to WT. Increases were insignificant only in 
young, i.e., sink leaves, probably provided by sulfate transport from mature leaves of E2, P2, and P3 Fig. (7).

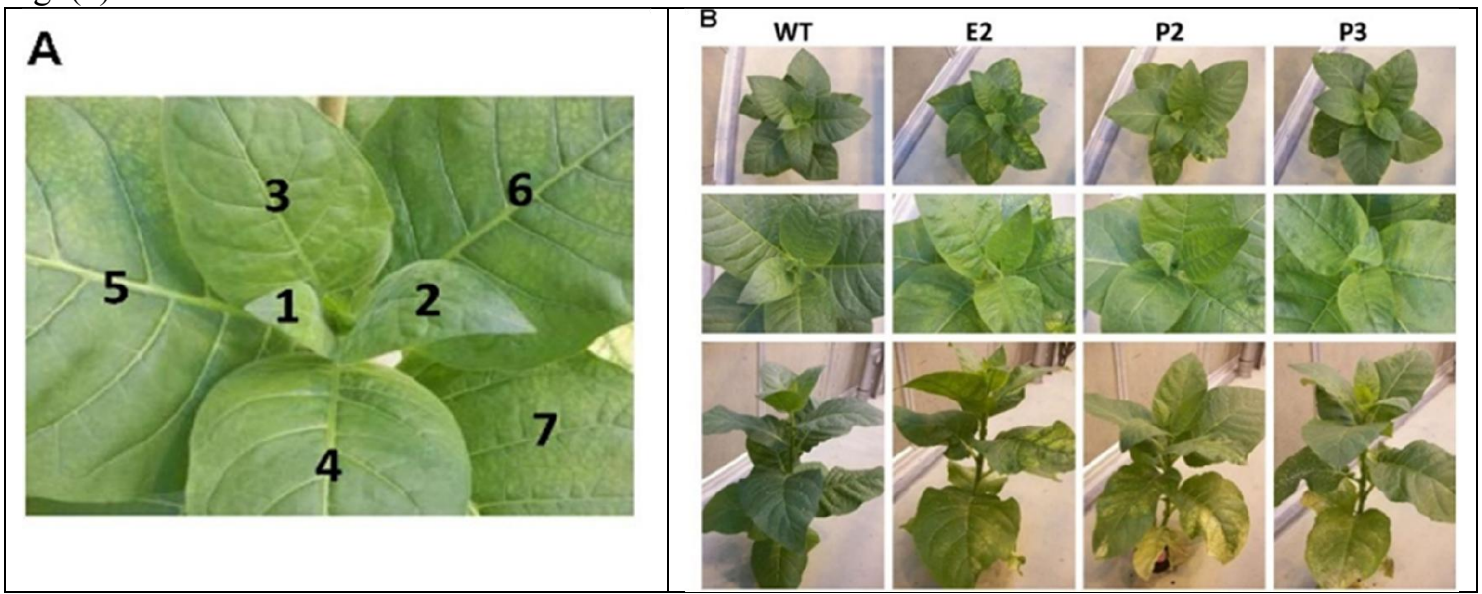

Fig. 9: Sulfite reductase (SIR) co-suppression tobacco lines.

$(A)$ Leaf numbering indicates the consecutive numbering of leaf samples for harvesting from youngest leaf $(\boldsymbol{L} 1)$ to oldest leaf harvested $(\boldsymbol{L} 7)$.

(B) Tobacco plants at the harvesting stage (approximately 2-month-old plant). Each panel shows a representative plant. Starting with $\boldsymbol{L} \boldsymbol{5}$ leaf chlorosis becomes visible. The phenotype actually resembles that of sulfate starvation as around vasculature tissue remains greener than between veins. In some cases, a retarded growth phenotype was observed. This $\boldsymbol{P} \mathbf{2}$ plant displays strong chlorosis and necrosis associated with older leaves after Marcell Nauman (2018).

Sulfate levels in tobacco control plants (WT) were strictly controlled between about 25 and 50 $\mu$ mol. $\mathrm{g}^{-1} \mathrm{FW}$ with slight increases toward older leaves. In lines E2, P2, and P3 this control is abrogated as sulfate accumulates with increasing leaf age, reaching between 75 and $100 \mu \mathrm{mol} / \mathrm{g} \mathrm{FW}$ in E2 and P2, and more than $150 \mu \mathrm{mol} . \mathrm{g}^{-1}$ activity can be assumed to result in a reduced flux to sulfide for cysteine biosynthesis. This pattern resembles that of sulfate starvation, despite of the fact that the transgenic plants over accumulate sulfate. This conclusion is supported by the fact that O-acetylserine (OAS) as the second precursor of cysteine biosynthesis next to sulfide tends to accumulate in E2, P2, and P3, though the increases were not as high as under sulfate starvation conditions. It might be speculated that the remaining SIR activity is just sufficient to provide sulfide for downstream synthesis of sulfur compounds to facilitate survival.. Homocysteine levels increased in leaves of mature WT tobacco plants. In E2, P2, and P3, homocysteine levels were comparably low in young leaves and remained low in older leaves displaying clear tendencies when comparing the medians. Methionine levels varied quite substantially and with no obvious pattern in WT leaves. The transgenic lines displayed reduced contents of methionine, especially in younger leaves. Sulfide contents, at $25-30 \mu \mathrm{mol} . \mathrm{g}^{-1} \mathrm{FW}$, were much higher in tobacco than reported for example for tomato (3-6 $\mu \mathrm{mol} / \mathrm{g}$ FW; Yarmolinsky et al., (2014), Arabidopsis approximately $10 \mu \mathrm{mol} / \mathrm{g} \mathrm{FW}$; Hubberten et al., (2012b). This puts an additional pressure on the transgenic plant lines, as tobacco seems to have a higher need for available sulfide. In summary, a picture emerges that sulfate, as an upstream component relative to the SIR bottleneck, accumulates. In parallel, thiosulfate accumulates, which is normally hardly detectable in tobacco leaves. Sulfite levels showed a tendency to increase, especially with increasing leaf age. Metabolites downstream of SIR displayed in SIR co-suppression lines a pattern resembling sulfate deprivation responses Nikiforova et al., (2005) while sulfide levels remained stable despite reduction of SIR activity.

As observed for many nutrient deficiency responses, the shoot/root ratio decreases during prolonged sulfate starvation. Interestingly, the morphological phenotype also includes changes in root architecture, where reactive oxygen species are produced in response to sulfate deprivation Schachtman and Shin (2007). The precise developmental response depends on the plant species, but in Arabidopsis, lateral root initiation is enhanced but not elongation Kutz et al. (2002). At least Arabidopsis roots are also able to grow toward sulfate-rich zones as has been observed for Arabidopsis and several crop plants in response to nitrate, phosphate or potassium rich patches of soil (Robinson (1994). Since sulfate 
belongs to the six essential plant nutrients, its availability has strong impact not only on plant growth and development, but also on crop yield and quality. Optimized sulfate fertilization that is in equilibrium with nitrogen application is of great concern in agriculture Hell and Hillebrand (2001); Howarth et al. (2008); Dubousset et al. (2009). Since the decline of atmospheric intake of $\mathrm{SO}_{2}$ due to clean air acts in the 1980s, the recognition of sulfate deficiency and precise fertilization regimes for sulfate has not only become a requirement in high yield agriculture, in particular for oilseed rape, but also cereals and sugar beet. Today, addition of $20-40 \mathrm{~kg} \mathrm{~S} / \mathrm{ha}$ are regular measures to optimize yield in sulfur deficient soils. The timing of application is important, for example, before the onset of seed filling, because, in marked difference to nitrogen metabolism, the current availability of stored sulfate and less its reactivation defines the availability to the seeds

Walker and Boothe (2003).

Naveen et al (2019), they reported that $¥$-Glutamylcyclotransferase initiates glutathione degradation to component amino acids L-glutamate, L-cysteine and L-glycine. The enzyme is encoded by three genes in Arabidopsis thaliana, one of which (GGCT2;1) is transcriptionally upregulated by starvation for the essential macronutrient sulfur (S). Regulation by S-starvation suggests that GGCT2;1 mobilizes L-cysteine from glutathione when there is insufficient sulfate for de novo L-cysteine synthesis. The response of wild-type seedlings to S-starvation was compared to ggct2;1 null mutants. S-starvation causes glutathione depletion in S-starved wild-type seedlings, but higher glutathione is maintained in the primary root tip than in other seedling tissues. Although GGCT2;1 is induced throughout seedlings, its expression is concentrated in the primary root tip where it activates the cglutamyl cycle. S-starved wild-type plants also produce longer primary roots, and lateral root growth is suppressed. While glutathione is also rapidly depleted in ggct2;1 null seedlings, much higher glutathione is maintained in the primary root tip compared to the wild type. S-starved ggct2;1 primary roots grow longer than the wild type, and lateral root growth Fig.( $8 \mathrm{a}, \mathrm{b})$.

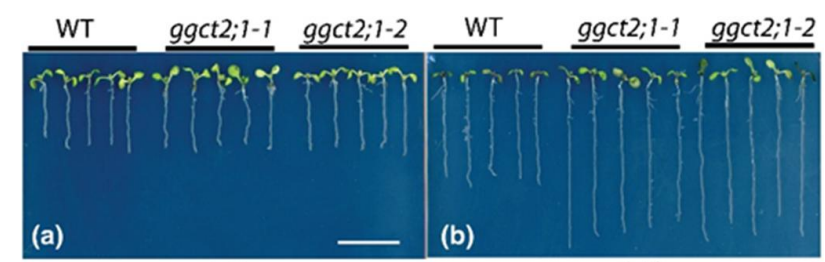

$(+S)$

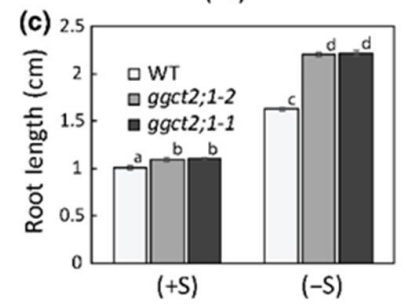

(-S)

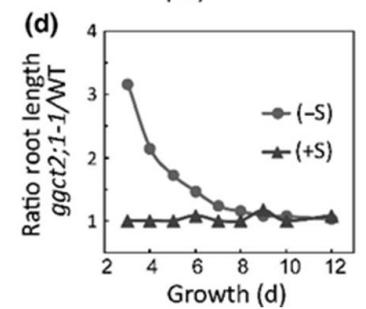

Fig.8a: Primary root growth phenotype of Arabidopsis ggct2;1 mutants. Arabidopsis seedlings were germinated and grown for $6 \mathrm{~d}$ on the indicated medium, and then photographed. (a) $+\mathrm{S}$ medium, (b) _S. Bar in (a), $1 \mathrm{~cm}$. (c) Primary root length. Values are the means of 25 seedlings SD. Values denoted with different letters are significantly different at $\mathrm{P}<0.01$. Root Length was measured over $12 \mathrm{~d}$ on $+\mathrm{S}$ and $\mathrm{S}$ and the ratio of ggct2;1-2 root length divided by wild-type (WT) root length was plotted (d). The values for ratio calculations were the means from 25 seedlings, 

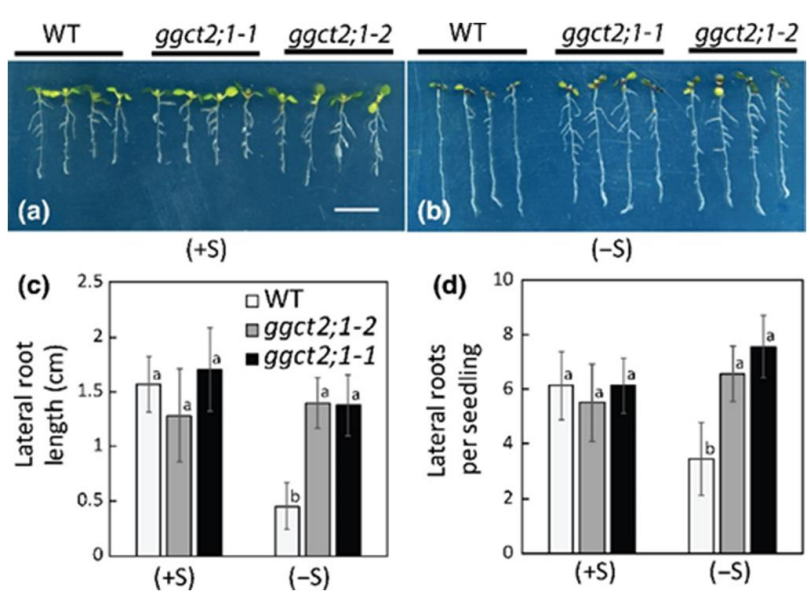

Fig. 8b: Lateral root phenotype of Arabidopsis ggct2;1 mutants. Arabidopsis Seedlings were germinated and grown for $9 \mathrm{~d}$ on the indicated medium, and then photographed. (a) $+\mathrm{S}$ medium, (b) S. Bar in (a), 1 $\mathrm{cm}$. (c) Total length of lateral roots; (d) number of visible lateral roots. Values are the means of nine seedlings SD. Values denoted with different letters are significantly different at $\mathrm{P}<0.01$. WT, Wild type. Naveen et al. (2019)

\section{3 - Methods of Sulfur uptake in soils}

\subsection{Gain of Sulfur through plant root systems}

Sulfate transporters are the most prominent group of S-metabolite transporters in plants because sulfate is the major source of sulfur taken up from the soil and because it is the most abundant Scontaining metabolite in plant cells. Accordingly, the first cloned gene for a transporter of sulfur metabolite in plants was a gene for SULTR Smith et al., (1995). In this pioneering work, the authors used the complementation of yeast mutant unable to take up sulfate to isolate three different cDNA clones for SULTRs from Stylosanthes hamate, a tropical forage legume. These cDNAs encoded two of high affinity transporters and one a low affinity transporter Smith et al., (1995). Functionally, these proteins are $\mathrm{H}+$ /sulfate co-transporters, which corresponds with their phylogenetic relation with SULTRs from other organisms. SULTRs can be divided into three major groups according to their mechanisms, ATP-dependent ABC type transporters, $\mathrm{Na}^{+}\left(\mathrm{H}^{+}\right)$/sulfate symporters, and sulfate/anion $\left(\mathrm{Cl}^{-}, \mathrm{CO}^{-2}\right.$, oxalate) antiporters Markovich and Aronson, (2007); Ohana et al., (2009); Takahashi et al., (2011a). The two latter groups are represented by the SLC13 and SLC26 gene families, respectively, Takahashi et al., (2011a). In higher plants, only the $\mathrm{H}+/$ SULTRs are present, while in green algae and many microalgae genes all three groups are present Takahashi et al., (2011a); Bochenek et al.,(2013). ABC-type of SULTRs are known to import sulfate to plastids of green algae but are not present in Bryophytes and seed plants Melis and Chen, 2005). Plant SULTRs are evolutionary related to the SLC26 group, but with a reaction mechanism of $\mathrm{H}+$ /sulfate symport, similar to the SLC13 group, which use Na+ Takahashi et al., (2011a). They are integrated into the membranes by 12 transmembrane regions and contain a STAS domain, found in SULTRs and with a significant similarity to bacterial anti-sigma factor antagonists Takahashi et al., $(2011 \mathrm{a}, \mathrm{b})$. The STAS domain is important for the correct incorporation into the membrane, activity, and interaction with other proteins Shibagaki and Grossman,( 2004), (2006). Already the first report of cloning of plant SULTRs demonstrated that multigene family Smith et al., (1995), encodes them. Arabidopsis possess 12 SULTR genes, whereas 11 genes are present in rice, 13 in poplar, and 5 SULTR genes are encoded in the sequenced genomes of basal plants Selaginella moellendorffii and Physcomitrella patens Kopriva et al., (2009); Takahashi et al., (2011a). The transporters can be divided in four distinct groups, which are also functionally divergent. The first group encodes high affinity SULTRs, group 2 are low affinity transporters, group 4 encodes vacuolar sulfate exporters, and the group 3 is the most diffuse from these groups, encoding transporters of the plastid membranes, symbiosome membranes, and others with specific or unknown functions Buchner et al., (2004b); Takahashi et al., (2011b). Every plant species possesses in addition one or two genes with a significant sequence similarity to SULTR, but lacking the STAS domain. These genes were traditionally included into the SULTR family as group 5, but since they were shown to be involved in 
transport of molybdate and could never be confirmed to transport sulfate TejadaJimenez et al., (2007; Tomatsu et al., (2007); Baxter et al., (2008), they are not considered to be SULTRs any more Takahashi et al., (2011a). The SULTR family is best characterized in Arabidopsis. Three genes form the group 1, SULTR1;1, and SULTR1;2 are expressed in roots and are responsible for sulfate uptake from the soil. Plants lacking both these transporters are unable to take up sulfate in low concentrations and are strongly affected in growth Yoshimoto et al., (2002), (2007); Rouached et al., (2008). The transporters have overlapping function, but are differentially regulated, with the SULTR1;1 playing an important role during sulfate starvation Rouached et al., (2008). On the other hand, during normal sulfate supply, SULTR1;2 is the more prominent transporter, as evidenced from the experiments showing selenate resistance of sultr 1;2 mutants Shibagaki et al., (2002). In addition, SULTR1;2 has been proposed to act as sensor of sulfur status of plants Zhang et al., (2014), but more evidence is necessary to dissect the mechanism of such sensing. SULTR $1 ; 3$ is a high affinity transporter localized in phloem and important for source-sink redistribution of sulfate Yoshimoto et al., (2003). The two low affinity group 2 transporters are localized in vasculature and are responsible for long distance translocation of sulfate Takahashi et al., (2000). Group 4 transporters are found in tonoplast and facilitate sulfate efflux from the vacuoles Kataoka et al., (2004b). The first group 3 transporter characterized was SULTR3;5, which was shown to modulate the function of SULTR2;1 but not to transport sulfate itself Kataoka et al., (2004a). The other members of this group were shown recently to be present in the plastid envelope and to catalyze sulfate import to the plastids Cao et al., (2013). Interestingly, all SULTR transcripts with exception of root specific SULTR1;1 were highly and coordinately enriched in bundle sheath cells of Arabidopsis leaves Aubry et al., (2014). Fig. (9) Shows the known (transporters 1-4) and yet to be identified SULTRs localized in various membranes within the plant cell.

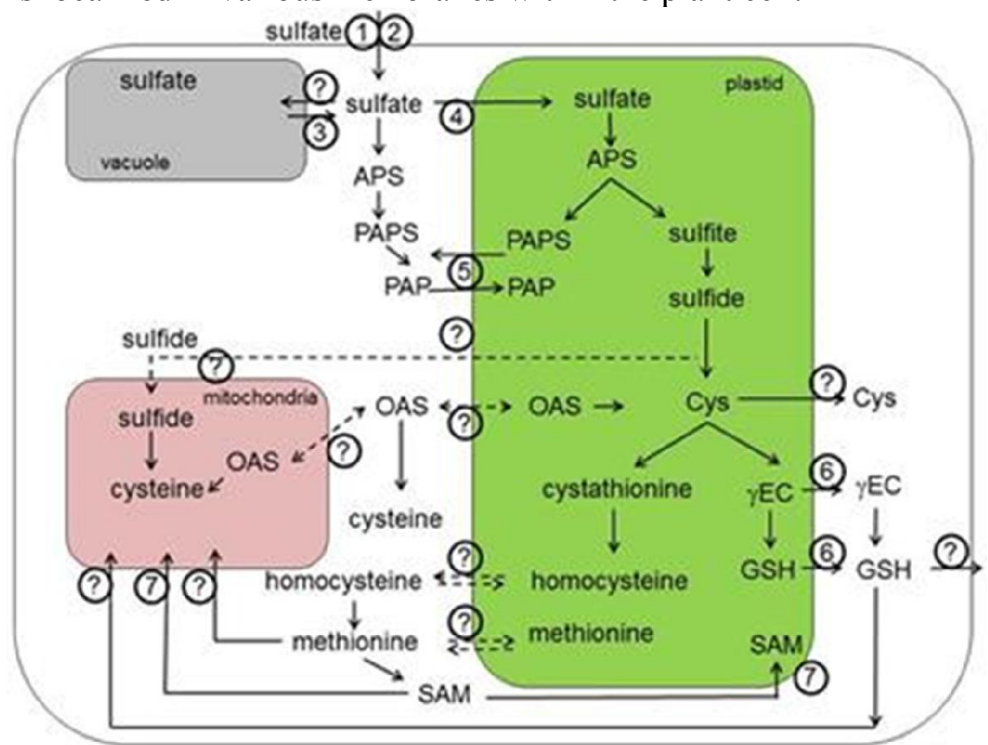

Fig. 9: Transport processes in primary sulfur assimilation after Tamara and Stanislav (2014)

Tamara and Stanislav (2014) reported that Sulfate is taken up by the root cells with the help of SULTR1;1 (1) and SULTR1;2 (2). Once crossed the plasma membrane of epidermal and cortical root cells, sulfate is transported through the series of sulfate transporters (SULTR) residing in various membranes within the plant. The SUTR4;1 and SULTR4;2 are important for the efflux from the vacuole into the cytoplasm (3). The transporter important for the sulfate influx into vacuole is still unknown. Import of sulfate into the chloroplasts is possible due to SULTR3;1 and probably other members of SULTR3 subfamily (4). PAPS is produced in both chloroplasts and the cytoplasm and can be exchanged between these compartments by PAPST/TAAC transporter (5). The known transporter of thiols (GSH and $\gamma \mathrm{EC}$ ) are chloroquine-resistance transporter (CRT)-like proteins or CRLs (6). However, an alternative transport system for thiols in the plastid membrane is expected to exist. In a similar way, the GSH transporters to the mitochondria await still the discovery. S-adenosylmethionine transporter 1 (SAMT1; 7) is a chloroplastidic protein involved in the exchange of SAM with Sadenosylhomocysteine, the by-product of methylation reactions that has to be regenerated to SAM in 
the cytoplasm. This is also the case for the plasmalemma-localized transporters of S-methylmethionine (SMM) and GSH, which are important transport form of reduced sulfur and therefore need to be exported out of the cell. APS, adenosine 5 -phosphosulfate; Cys, cysteine; OAS, O-acetylserine; $\gamma \mathrm{EC}$, $\gamma$-glutamylcysteine; GSH, glutathione; SAM, S-adenosylmethionine; PAPS, 3 -phosphoadenosine-5 phosphosulfate; PAP, 3 -phosphoadenosine 5 -phosphate. Dashed lines indicate theoretically possible transport pathways.

\section{3-2 Order of Sulfate Transporter}

Sulfate uptake plays a major role in the control of plant sulfur homeostasis Vauclare et al., (2002). This is evident from the response of sulfate uptake to sulfur availability and its regulation by environmental conditions. Sulfate uptake is induced under sulfate limiting conditions and is repressed in the presence of reduced sulfur Smith et al., (1995), (1997); Takahashi et al., (1997), (2011b); Yoshimoto et al., (2002). Sulfate uptake is coordinated with the uptake of nitrate, so at low nitrate levels and with availability of carbohydrates sulfate uptake is repressed (Smith, (1980; Brunold, (1993); Koprivova et al., (2000); Kopriva et al., (2002). Particular significance for overall control of plant sulfur nutrition has regulation by the precursor of Cys, Smith et al., (1997); Hopkins et al., (2005). Many experiments showed that the regulation of sulfate uptake is well correlated with regulation of mRNA levels of the transporters, in particular those of group 1 (Smith et al., (1997); Takahashi et al., (1997),( 2011b); Abola et al., (1999); Yoshimoto et al., (2002); Rouached et al., (2008). Indeed, sulfate starvation, which results in increased sulfate uptake, induces transcript levels of Arabidopsis SULTR1;1, 1;2, 2;1, 4;1, and 4;2 (Takahashi et al., (1997), (2000); Abola et al., (1999); Yoshimoto et al., (2002); Kataoka et al., (2004b). The same is true for other plant species, increase of SULTR transcript levels was observed in sulfur starved Brassica, wheat, Medicago, barley, etc. (Smith et al., (1997); Abola et al., (1999); Buchner et al., (2004a), (2010); Koralewska et al., (2009); Casieri et al., (2012). OAS, which accumulates in sulfur starved plants, induces mRNA levels of SULTR genes even at sufficient sulfate supply (Smith et al., (1997); Hirai et al., (2003); Hopkins et al., (2005), and may so act as a signal in the sulfate starvation regulatory network (Hirai et al., (2005); Hubberten et al., (2012). The transcript levels of SULTR are rapidly reduced when sulfate is resupplied to sulfur starved plants Koralewska et al., (2009). Sulfate starvation is one of the best-analyzed environmental condition using systems biology approaches (Hirai et al., (2003); Maruyama-Nakashita et al., (2003); Nikiforova et al., (2003), (2004); Hubberten et al., (2012). The efforts to dissect the mechanisms of the regulation resulted in identification of at least some components of the regulatory circuits. One Cis and one Tran's factor important for the regulation of SULTR genes have been identified. Analysis of promoter of SULTR $1 ; 1$ gene revealed a presence of a 16-bp SURE, present in promoters of many S-starvation inducible genes (Maruyama-Nakashita et al., (2005). The transcription factor SLIM1 has been identified by a genetic screen using SULTR1;2::GFP as reporter construct (MaruyamaNakashita et al., (2006). SLIM1 is a member of the EIL family transcription factors, ETHYLENE-INSENSITIVE3-LIKE3 (EIL3), and controls the sulfate starvation response of a large number of, but not all, responsive genes (MaruyamaNakashita et al., (2006); Kawashima et al., (2011). Since SLIM1 mRNA is not affected by S-starvation, the mechanisms of its action is not known, it is also not clear whether it actually binds to the SURE element. However, experiments with phosphatase inhibitors revealed that dephosphorylation is a part of the signal transduction MaruyamaNakashita et al., (2004a) and that cytokinins may be important for controlling SULTR1;1 expression Maruyama-Nakashita et al., (2004b). The regulation of sulfate uptake is, however, more complex and includes post-transcriptional mechanisms. In sultr1;1 sultr1;2 plants complemented by constitutively expressed SULTR $1 ; 1$ and $1 ; 2$, accumulation of the transporters was induced by S-starvation despite the mRNA accumulation being not affected Yoshimoto et al., (2007). Thus, the induction of sulfate uptake is not completely derived from upregulation of the transcript but another, unknown, post-transcriptional mechanism is necessary for proper regulation. Another posttranscriptional mechanism to improve sulfate uptake during S-starvation affects different SULTR isoform, the low affinity SULTR2;1. The SULTR2;1 is a target of microRNA miR395, which is induced by S-starvation in a SLIM1- dependent manner Kawashima et al., (2009), (2011). The function of SULTR2;1 in response to S-starvation is to increase the translocation of sulfate from the roots to the shoots. The mechanism of the miR395 regulation of SULTR2;1 is, however, non-canonical, as both the miRNA and its target are actually induced by S-starvation. The spatial expression patterns of the two transcripts are no overlapping, so that miR395 restricts SULTR2,1 expression to the xylem parenchyma 
cells and prevents its accumulation in phloem companion cells. This expression pattern of SULTR2;1 increases the efficiency of xylem loading, to increase translocation of sulfate to the leaves and prevents the unloading from phloem back to the roots Kawashima et al., (2009) Fig.(10).

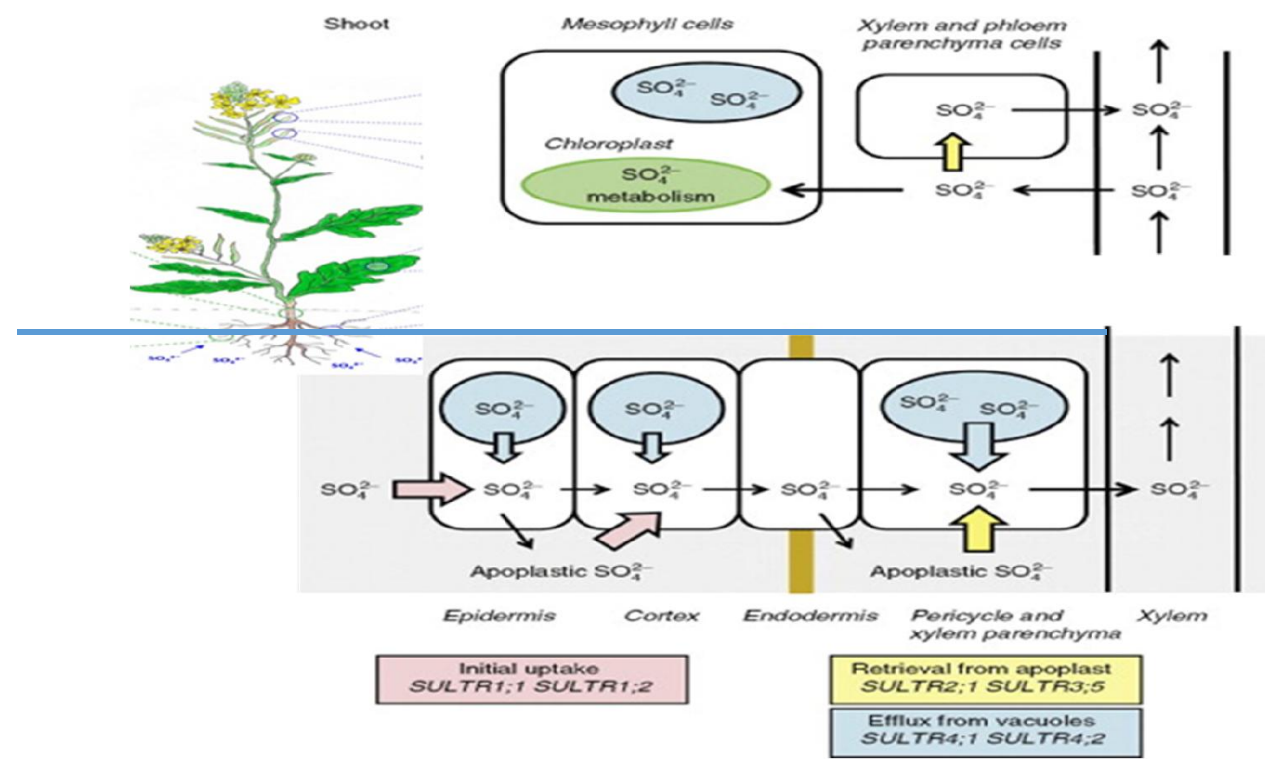

Fig. 10: Diagram represented regulation of sulfate to the assimilation in plant

The third post-translational mechanism of regulation of sulfate uptake is the modulation of SULTR1;2 activity through interaction with the Cys synthase in cytoplasm. The Cys synthase binds to the STAS domain and reduces the activity of the transporter; at the same time the interaction results in increased activity of the Cys synthase as shown in fig.(9) Shibagaki and Grossman, (2010). Despite the impressive progress in understanding of plant sulfate transport, and the recent identification of longsought plastidic SULTR Cao et al., (2013), many questions remain open. The transporters responsible for sulfate influx into vacuoles are still not known. The redundancy of the plastidic transporters SULTR3;1- 3;4 is in contrast to the specific functions of the other SULTR genes and a detailed dissection of their individual function still has to be undertaken. Given the subtle effects of sultr3 mutants on seeds Zuber et al., (2010), this might not be a trivial task. The possible function of SULTR1;2 (Zhang et al., (2014) as a sulfate sensor is intriguing, particularly as the Chlamydomonas regulator of S-starvation response, SAC1, is similar to SULTR of the SLC13 group Davies et al., (1996); Takahashi et al., (2011a). The molecular mechanisms of regulation of SULTR need to be elucidated; apart from SLIM1 and HY5, no other transcription factors binding the SULTR1;2 promoters have been reported Maruyama-Nakashita et al., (2006); Lee et al., (2011). Sulfate transport will thus further remain in prime focus of investigations of plant sulfur metabolism.

\section{Transport of Cysteine and Glutathione}

Intracellular transport of amino acids is essential, as in plants protein synthesis occurs in three organelles. In has been hypothesized, however, that this may not to be the case for Cys, as Cys synthesis is also localized in these three compartments, cytosol, mitochondria, and plastids Lunn et al., (1990), Fig. (11). However, analysis of mutants in the OASTL revealed that the Cys synthesis could be restricted to a single compartment without affecting survival and with only small effects on growth Heeg et al., (2008); Watanabe et al., (2008); Birke et al., (2012). Thus, Cys or its precursor must be transported across the mitochondrial and plastid membranes in both directions. Investigations of Cys transport across mitochondrial membrane revealed presence of multiple transport systems with different kinetic properties Lee et al., (2014), but specific transporters have not been reported in plants. Cys also undergoes intercellular transport, although its contribution to a total long-distance flow of sulfur may not be very high Herschbach and Rennenberg, (1995). 


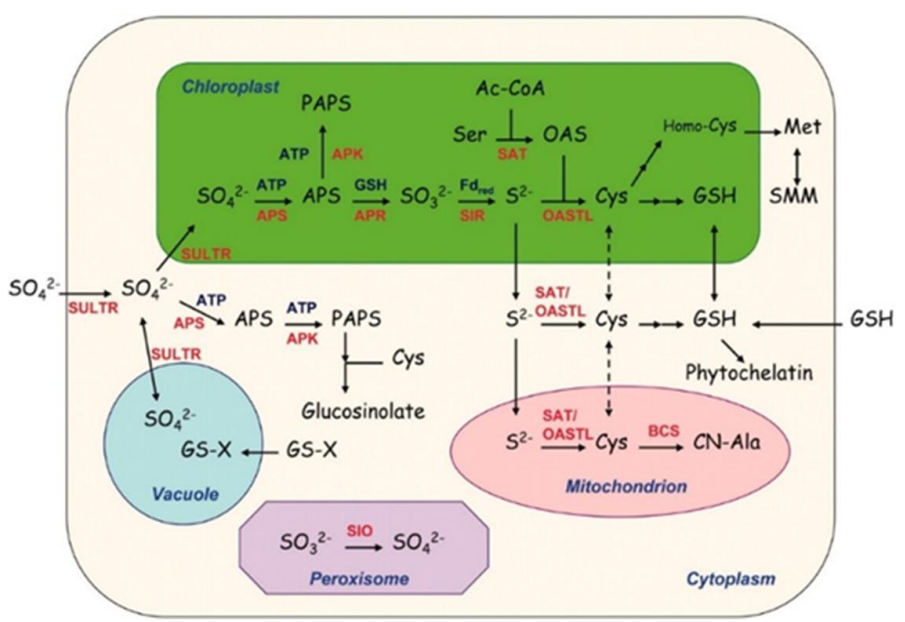

Fig.11: Sulfur assimilatory metabolism in the subcellular compartments of plant cells. Black indicates name of metabolites: Ac-CoA, acetyl-CoA; APS, adenosine 5\#-phosphosulfate; CN-Ala, b-cyano-Ala; GSH, reduced glutathione; GS-X, glutathione conjugate; OAS, O-acetyl-Ser; PAPS, 3\#-phosphoadenosine-5\#phosphosulfate (3\#-phosphoadenylylsulfate); SMM, S-methyl- Met. Blue indicates names of cofactors: GSH, reduced glutathione; Fdred, reduced ferredoxin. Red indicates the name of proteins: APK, APS kinase; APR, adenosine 5\#-phosphosulfate reductase; APS, ATP sulfurylase; BCS, b-cyano-Ala synthase; OASTL, OAS(thiol)lyase; SAT, Ser acetyltransferase; SIO, sulfite oxidase; SIR, sulfite reductase; SULTR, sulfate transporter

For example, seeds are able to assimilate sulfate and are therefore not dependent on transport of Cys Tabe and Droux, (2001). In C4 plants, however, sulfur nutrition is dependent on intercellular Cys transport, since sulfate is reduced in the bundle sheath cells only and Cys is the transport metabolite from these cells to mesophyll and other cell types of the leaves Burgener et al., (1998); Kopriva and Koprivova, (2005). Plants possess a large number of amino acid transporters, many of them capable of transporting Cys, some even with a high specificity Miranda et al., (2001); Tegeder, (2012). In yeast, Cys can be transported by at least eight unspecific amino acid permeases, but the major contributor is a specific YCT1 Kaur and Bachhawat, (2007), De Kraker (2011). In animals, multiple systems transport Cys rather than Cys McBean and Flynn, (2001). It is not clear whether similar specific Cys (or Met) transporters exist in plants or whether Cys transport is less specific through general amino acid permeases. Thus, the molecular nature of Cys transport into the cells as well as in mitochondria and plastid membranes remains to be elucidated. Cys as the first product of sulfate assimilation is used in many metabolic processes. Among the most important sulfur compounds in plant cells derived from Cys is the tripeptide GSH, $\gamma$-glutamylcysteinyl glycine Noctor et al., (2012). GSH is synthesized in two steps from the constituting amino acids, but in this synthesis, a transport step is of utmost importance. In contrast to initial reports placing GSH synthesis to both plastids and cytoplasm, the first enzyme of the pathway, $\gamma$-glutamylcysteine synthetase, is strictly localized in plastids, at least in Arabidopsis Wachter et al., (2005). GSH synthetase, on the other hand is present in both compartments but mostly in the cytoplasm. The intermediate $\gamma$-glutamylcysteine thus has to be exported from the plastids for efficient GSH synthesis Fig. (12)

This conclusion has been confirmed by showing that cytosolic expression of GSH synthase rescues the seedling lethal gsh2 mutant Pasternak et al., (2008). GSH itself is present in all compartments, with particularly high concentration in the mitochondria Zechmann et al., (2008). Thus, mitochondrial GSH transporter has to be postulated for plant cells Fig. (9). In addition, GSH is one of the forms of reduced sulfur subjected to long-distance transport Herschbach and Rennenberg, (1995), with the need for plasma membrane transporters. 


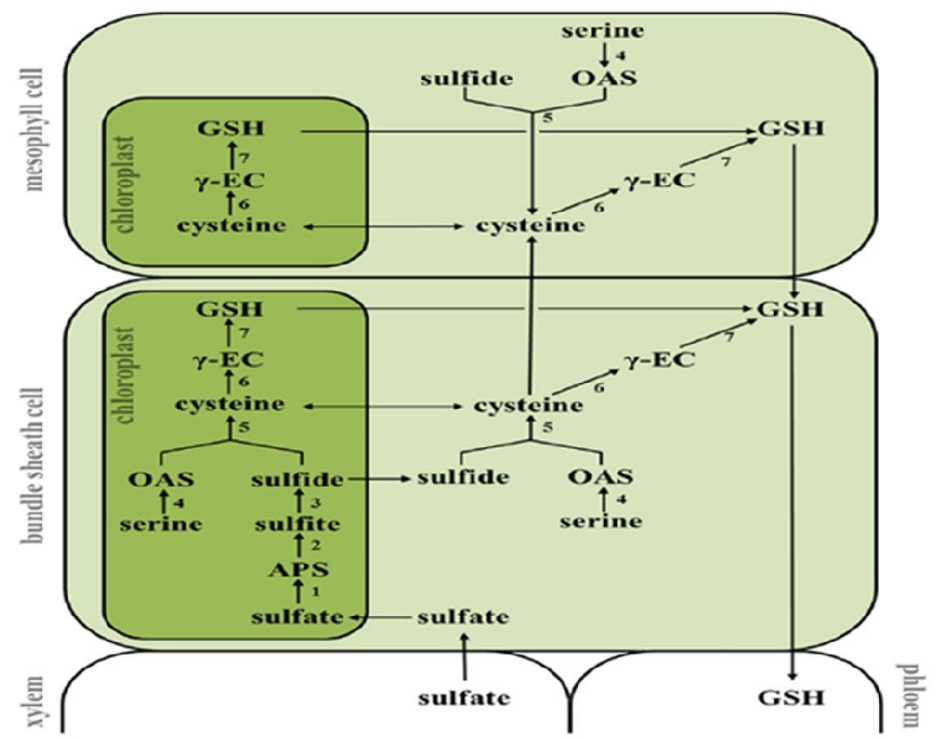

Fig. 12: Illustrates intercellular compartmentation of Sulfate Assimilation and glutathione biosynthesis in maize. After Silke, and Stanislav (2015)

Sulfate is taken up from the soil and transported to the bundle sheath cells (BSC) through the xylem. The reduction of sulfate takes place exclusively in the plastids of BSC and is mediated by ATP sulfurylase (1), APS reductase (2), and sulfite reductase (3). Sulfide is further incorporated into the amino acid backbone of OAS-by-OAS (thiol) lyase (5) to form cysteine in chloroplasts, cytosol and mitochondria (not included) of BSC. OAS is derived from serine by serine acetyltransferase-mediated acetylation (4). Reduced sulfur is transported in form of cysteine to mesophyll cells where glutathione (GSH) synthesis is predominantly localized. GSH synthesis is driven by $\gamma$-EC synthetase (6) and GSH synthetase (7). APS, adenosine-5'-phosphosulfate; GSH, glutathione; OAS, O-acetylserine; $\gamma$-EC, $\gamma$ glutamylcysteine. While the presence of such GSH transporters was long recognized, the molecular nature of such carriers is still far from being determined. The identification of high affinity GSH transporter in yeast Bourbouloux et al., (2000) triggered the search for plant GSH transporter, particularly as Arabidopsis possess nine homologs of the yeast HGT1 Cagnac et al., (2004). While some transporters of the oligopeptide transporter family indeed transported GSH Bogs et al., (2003); Cagnac et al., (2004); Zhang et al., (2004), Amir et al. (2002) the affinity and specificity was not as high as expected for the high flux of GSH within plant cells. An alternative pathway for GSH transport has been proposed, analogical to the animal gamma-glutamyl cycle, in which GSH is moved across the membrane through combination of degradation, amino acid transport, and synthesis Meister et al., (1981).

This conclusion has been confirmed by showing that cytosolic expression of GSH synthase rescues the seedling lethal gsh2 mutant Pasternak et al., (2008). GSH itself is present in all compartments, with particularly high concentration in the mitochondria Zechmann et al., (2008). Thus, mitochondrial GSH transporter has to be postulated for plant cells. In addition, GSH is one of the forms of reduced sulfur subjected to long-distance transport Herschbach and Rennenberg, (1995), with the need for plasma membrane transporters. While the presence of such GSH transporters was long recognized, the molecular nature of such carriers is still far from being determined. The identification of high affinity GSH transporter in yeast Bourbouloux et al., (2000) triggered the search for plant GSH transporter, particularly as Arabidopsis possess nine homologs of the yeast HGT1 Cagnac et al., (2004). While some transporters of the oligopeptide transporter family indeed transported GSH Bogs et al., (2003; Cagnac et al., (2004); Zhang et al., (2004), the affinity and specificity was not as high as expected for the high flux of GSH within plant cells. An alternative pathway for GSH transport has been proposed, analogical to the animal gamma-glutamyl cycle, in which GSH is moved across the membrane through combination of degradation, amino acid transport, and synthesis Meister et al., (1981). The key enzyme of this cycle, the GGT is present in plants and has been shown to be important for recovery of apoplastic GSH Ferretti et al., (2009), its contribution to total GSH uptake has, however, 
not been clarified. Since GGT is localized on the apoplast side of plasma membrane or in the tonoplast, it cannot be responsible for the intracellular GSH transport Fig. (13) The first step to understanding the molecular nature of GSH movement between the organelles was identification in Arabidopsis of a small family of genes similar to chloroquine-resistance transporter of Plasmodium falciparum Maughan et al., (2010). These CLT were found in a genetic screen using the effect of inhibition of GSH synthesis on root growth Koprivova et al., (2010); Maughan et al., (2010).

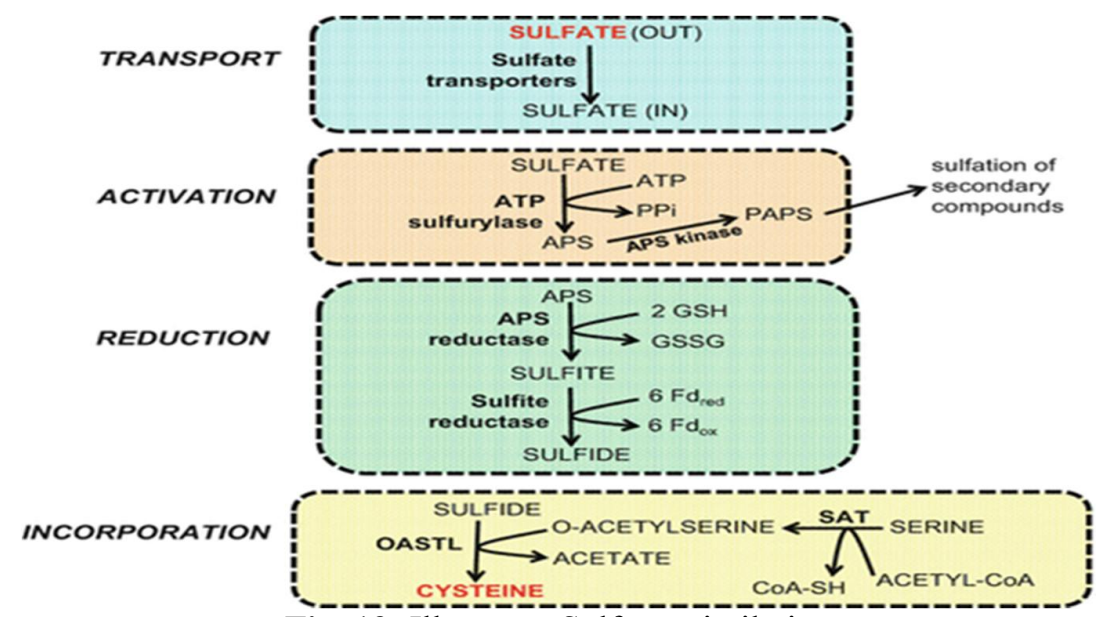

Fig. 13: Illustrates Sulfur assimilation

The first step to understanding the molecular nature of GSH movement between the organelles was identification in Arabidopsis of a small family of genes similar to chloroquine-resistance transporter of Plasmodium falciparum Maughan et al., (2010). These CLT were found in a genetic screen using the effect of inhibition of GSH synthesis on root growth Koprivova et al., (2010); Maughan et al., (2010). All three CLTs are found in chloroplast membranes and affect the distribution of GSH between plastids and cytoplasm. They facilitate transport of GSH and $\gamma$-EC, probably as export from the plastids Maughan et al., (2010). Loss of CLTs results in sensitivity to cadmium, increased susceptibility to Phytophora, and affects root architecture Maughan et al., (2010); Schnaubelt et al., (2013). However, Arabidopsis mutants devoid of all three CLTs are viable, so alternative transport systems for thiols in the plastid membrane have to exist. Similarly, GSH transporters to the mitochondria await discovery. Glutathione is not only the most important redox buffer, it is also the precursor for synthesis of PC, small peptides involved in binding and detoxification of heavy metals Vatamaniuk et al., (1999). Since it had been long known that the last step of detoxification is the transport of the PC-metal complexes into the vacuole, the corresponding transporters have long been sought for Hall, (2002). In fission yeast, an ABC transporter present in the tonoplast is responsible for such transport and its loss results in cadmium sensitivity Ortiz et al., (1992). Plant possess a large number of $\mathrm{ABC}$ transporters with potentially the same function, therefore, first plant $\mathrm{ABC}$ transporters, importing PC-As complexes into the vacuole, have been identified only recently Song et al., (2010). Loss of two ABC transporters, AtABCC1 and AtABCC2, results in reduced arsenic tolerance, and on the other hand, their expression in yeast increases as tolerance. The AtABCC1 and AtABCC2 are involved also in detoxification of cadmium and mercury Park et al., (2012). The identification of these transporters is particularly important for engineering heavy metal tolerant and perhaps phytoextracting organisms. PCs are, however, also important for long-distance transport of metals, it remains to be shown, whether the same or other ABC transporter participate in such transport.

\section{Transport of Methionine and its Derivatives}

Methionine is another S-containing amino acid with a complex demand for inter- and intracellular transfer, particularly when its derivatives are taking into account. Similar to Cys, Met has to be present, and/or transported, to all compartments with protein synthesis Fig. (14). 


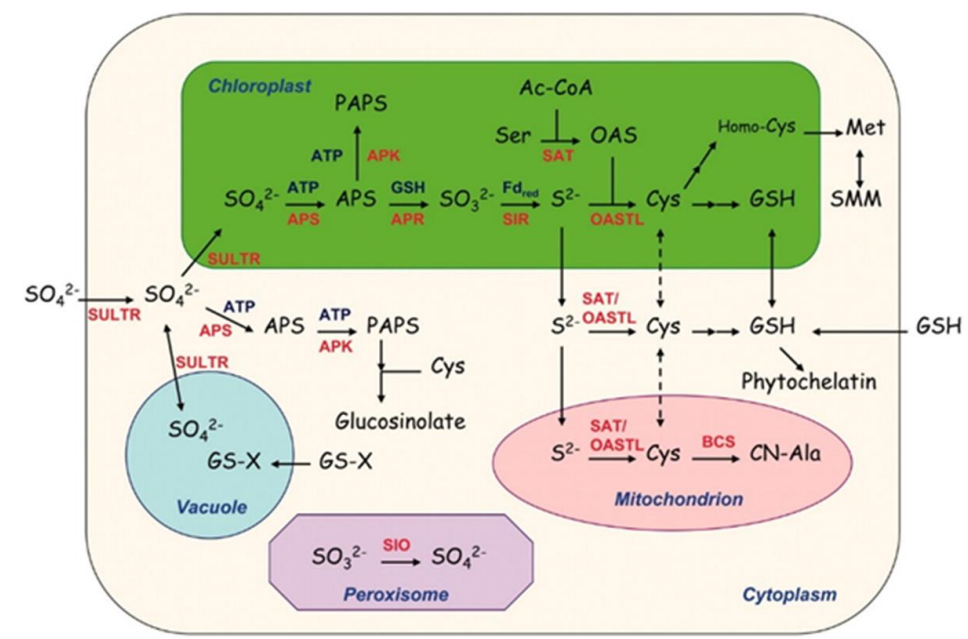

Fig. 14: Sulfur assimilatory metabolism in the subcellular compartments of plant cells. Black indicates name of metabolites: Ac-CoA, acetyl-CoA; APS, adenosine 5\#-phosphosulfate; CN-Ala, b-cyano-Ala; GSH, reduced glutathione; GS-X, glutathione conjugate; OAS, O-acetyl-Ser; PAPS, 3\#-phosphoadenosine-5\#-phosphosulfate (3\#phosphoadenylylsulfate); SMM, S-methyl-Met. Blue indicates names of cofactors: GSH, reduced glutathione; Fdred, reduced ferredoxin. Red indicates the name of proteins:APK, APS kinase; APR, adenosine 5\#-phosphosulfate reductase; APS, ATP sulfurylase; BCS, b-cyano-Ala synthase; OASTL,OAS(thiol)-lyase; SAT, Ser acetyltransferase; SIO, sulfite oxidase; SIR, sulfite reductase; SULTR, sulfate transporter. After Kazuki Saito (2004)

In addition, traditionally it was thought that Met synthesis in plants requires transport of homocysteine from plastids to the cytoplasm; as the activity of the last enzyme of the biosynthetic pathway, MS, was found in this compartment only Eichel et al., (1995). However, a plastid localized MS has been described leading to the conclusion that plastids are autonomous for Met synthesis and that cytosolic MS is involved predominantly in regeneration of Met for SAM synthesis in the SAM cycle Ravanel et al., (2004); Sauter et al., 2013) and for the biosynthesis of Met-derived GSL Schuster et al., (2006). However, interestingly, no reports on the importance of the plastidial MS and the possibility of complementation of its function by the cytosolic isoform are available. SAM is a methyl donor and cofactor in numerous cellular processes and its synthesis uses some $80 \%$ of newly synthesized Met Hesse et al., (2004). SAM is required in all organelles and has to be transported across plastidic and mitochondrial membranes, since it is synthesized only in the cytoplasm Wallsgrove et al., (1983); Shen et al., (2002). The SAM transporter (SAMT) has been identified in Arabidopsis by Bouvier et al. (2006) This transporter, similar to yeast mitochondrial SAM carrier, was shown to be additionally localized in mitochondria, and to contains five transmembrane helices Bouvier et al., (2006); Palmieri et al., 2006). The SAMT1 catalyzes exchange of SAM with S-adenosylhomocysteine, the byproduct of methylation reactions that has to be regenerated to SAM in the cytoplasm. SAMT1 is important, but not essential, as demonstrated by survival and setting seeds, but strong morphological phenotypes of the corresponding T-DNA line Bouvier et al., (2006). Another Met derivative, SMM is found in the phloem as an important transport form of reduced sulfur Bourgis et al., (1999); Fig. (15). Plant SMM transporters are not known, but expression of a yeast SMM transporter in pea resulted in improved sulfur and nitrogen content of the seeds Tan et al., (2010). The yeast SMM transporter is distinct from the SAMT described above and does not have clear homologs in plants Rouillon et al., (1999). How is SMM unloaded into the phloem in plants, therefore, still needs to be clarified. CHLOROPLASTIDIC TRANSPORTER IN THE BIOSYNTHESIS OF MET-DERIVED GSLs Glucosinolates are a group of amino acid derived secondary compounds, important for plant defense against various pests but also for human nutrition as determinants of taste and smell of crucifers and because of their anticancer genic properties Sonderby et al., (2010). 


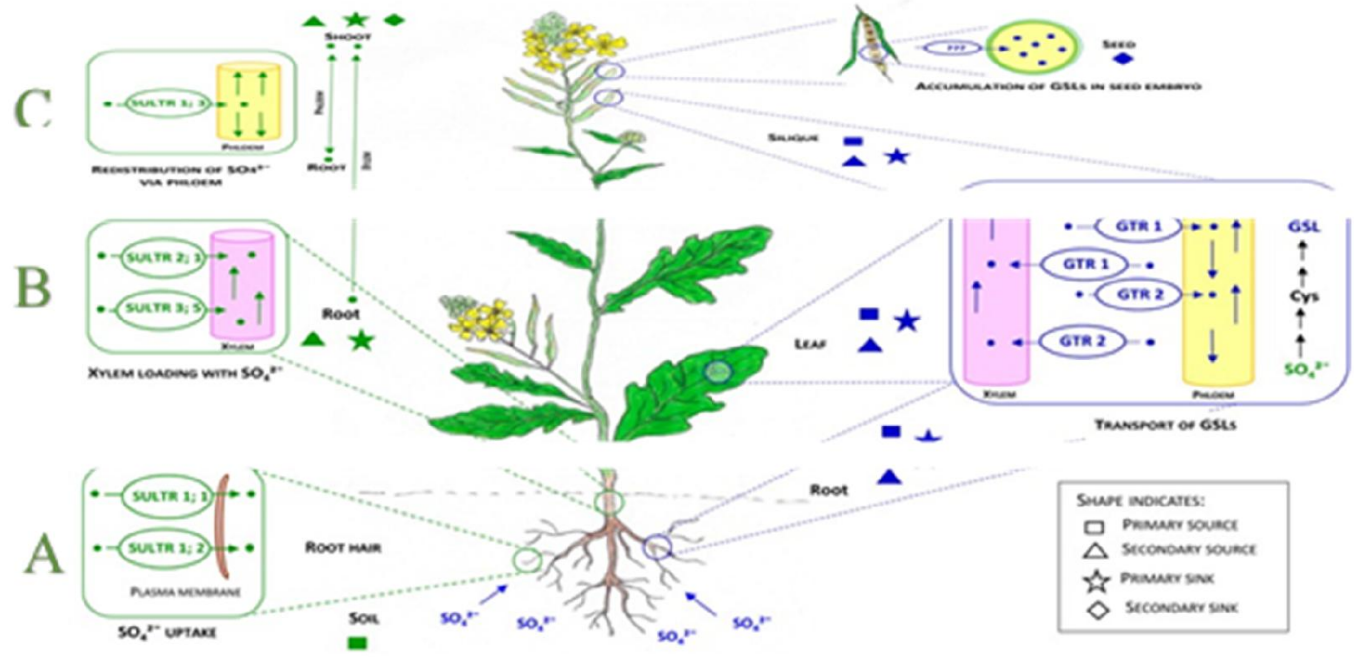

Fig. 15: Long distance transport in sulfur metabolism. Sulfate is taken up by the roots by SULTR1;1 and SULTR1;2. Root-to-shoot transfer of sulfate is facilitated by SULTR2;1 and SULTR3;5 Transport of sulfate to phloem is possible due to SULTR1;3 are transporters of GLS known as GTRs (glucosinolate transporters). They mediate the bidirectional long distance transport of GLS between roots and shoots via both phloem and xylem; facilitate the phloem loading, transport of GSL from the source to the sink tissue and import of GLS into the seeds. S-methylmethionine (SMM) and GSH are also important transport form of reduced sulfur found in phloem. How SMM and GSH are loaded into the phloem in plants, remains now unknown. The long distance transporters of flavonoids have been also predicted to exist but still await an identification. After Tamara and Stanislav (2014)

Enzymes required for the biosynthesis of GSLs core structure have been identified and shown to be localized in cytoplasmic compartment. In brief, the amino acid is converted to aldoximes by CYP79Fs converting Met derivatives Hansen et al., (2001); Chen et al., (2003). Next, aldoximes are oxidized to either nitrile oxides or aci-nitro compounds with CYP83A1 Bak and Feyereisen, (2001); Hemm et al., (2003); Naur et al., (2003). Following fusion of the activated aldoximes to GSH, the produced S-alkylthiohydroximates are converted to thiohydroximates by the C-S lyase SUR1 Mikkelsen et al., (2004). The last but one reaction in GSL biosynthesis is the S-glycosylation of thiohydroximates by glucosyltransferases of the UGT74 family, with UGT74C1 recently shown to glucosylateMet-derived substrates Douglas Grubb et al., (2014) Fig. (16).

The final step of GSL biosynthesis is a SOTs, which form GSL from desulfoGSL. PAPS made by APS kinase mainly in chloroplasts Mugford et al., (2009) provide the sulfate for the SOT reaction. Met is an essential constituent for the production of aliphatic GSLs in Arabidopsis thaliana. Before entering the GSL core biosynthetic pathway, Met undergoes chain elongation process in chloroplasts, which is similar to the conversion of the branched chain amino acid Val to Leu in primary metabolism. This process and its linkage with cytosolic GSL synthesis requires two transport steps across the chloroplast envelope, as the first reaction allowing Met to enter biosynthesis of GSLs is a deamination of Met by a cytosolic BCAT4 Schuster et al., (2006). Three following reaction are known to take place in chloroplasts, where the respective 2-keto-acid derived from MTOB needs to be imported. The MTOB-transporter was identified as BAT5 or BASS5 following its activation in trans by R2R3 MYB factors regulating GSL biosynthesis Gigolashvili et al., (2009). Notably, the reduction of BAT5 transcripts in bat5 mutant resulted in up to $50 \%$ decreased levels of Met-derived GSLs, indicating a role of BAT5 in the transport of GS intermediates across the plastid envelope Gigolashvili et al., (2009); Sawada et al., (2009b). Based on the analysis of bat5, its metabolic complementation by 2-keto-acids and genetic complementation using chemically inducible promoter fused to BAT5 CDS, the BAT5 has been proposed as a facilitator of keto-acids across chloroplasts Gigolashvili et al., (2009); Sawada et al., (2009b). Interestingly, not only MTOB, but also other 2-keto acids like 5-methylthio-2oxopentanoate (MTOP); 6-methylthio-2-oxohexanoate (MTOH), and 7-methylthio-2-oxoheptanoate seem to be exchanged between plastid and cytoplasm by BAT5. Indeed, feeding of wild-type and bat5 roots of Arabidopsis with 2-keto acids with different chain-length demonstrated that they are substrates 
of BAT5 since they could be successfully imported into chloroplasts of wild-type plants but not in the bat5 plants.

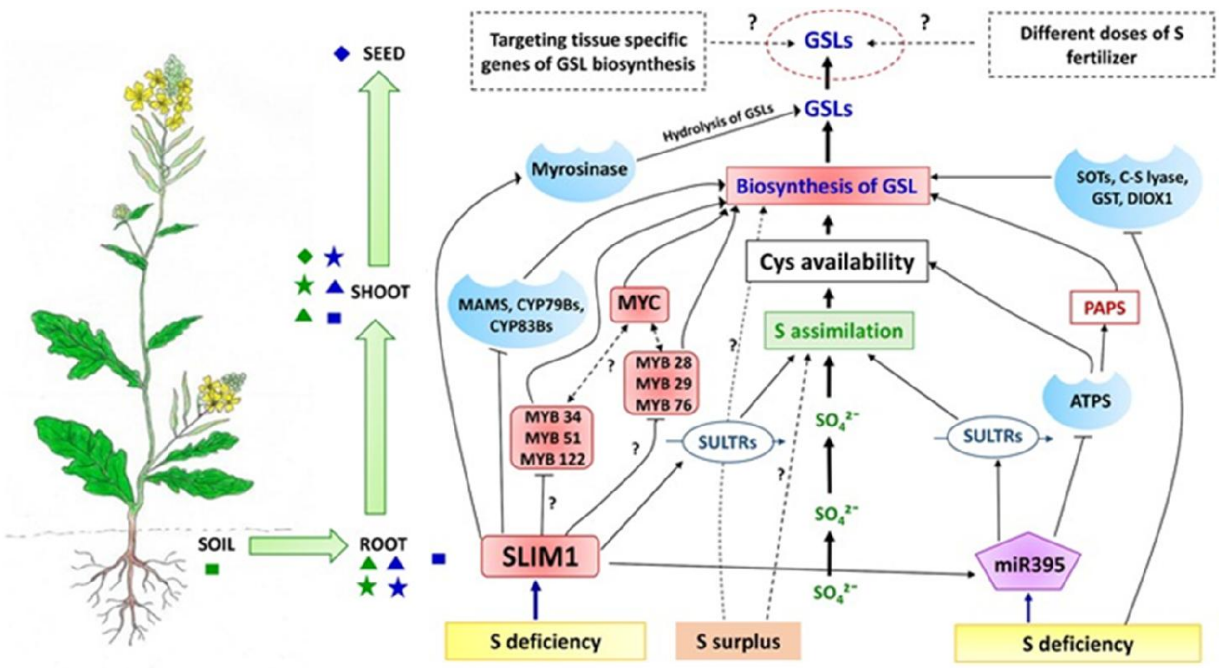

Fig. 16: A conceptual model how soil S status affects GSL accumulation in Brassica crops. Under S deficiency, the SLIM1 TF can down regulate the expression of key GSL biosynthesis enzymes (MAMS, CYP79Bs, and CYP83Bs) and MYB TFs (Lewandowska and Sirko, 2008; Frerigmann and Gigolashvili, 2014b). Further investigation needed to understand the complete picture of regulation of MYBs targeting indole GSL biosynthesis (MYB34, MYB51, MYB 122) and aliphatic GSL synthesis (MYB28, MYB 29, MYB76) by SLIM1 under different $\mathrm{S}$ conditions. In contrast, under S deficient conditions SLIM1 can also up regulate the expression of SULTRs and miR395, as well as the GSL hydrolysis enzyme (myrosinase) (Takahashi et al., 2011). In addition, upregulation of miR395 via SLIM1 under S deficiency can increase the expression of SULTRs and lead to enhanced uptake of sulfate from soil. SLIM1 can also down-regulate the expression of ATPS in the first step of S assimilation (Liang et al., 2010) which can affect the availability of Cys and PAPS for GSL biosynthesis. Sulfur deficiency has been suggested as a negative regulator of SOTs, C-S lyases, GST and AOPs enzymes of the GSL biosynthesis pathway (Nikiforova et al., 2005), although additional signaling molecules may be involved in this regulation. Moreover, we would expect the presence of additional signaling molecules and TFs, which can regulate both systems ( $\mathrm{S}$ assimilation and GSL biosynthesis) under S deficiency or varying S levels of the primary source (soil). As seeds are the ultimate sink for GSLs, optimizing the external application of S fertilizer and identifying the role of tissue-specific genes of GSL biosynthesis under different S levels would provide scope for manipulation of seed GSL levels in brassicas. Primary and secondary sources and sinks for S and GSLs are shown as indicated as in Figure (15). Shapes in green indicate sources and sinks related to the sulfate system whereas shapes in blue indicate the GSL system. Rectangular shapes indicate primary sources, triangles indicate secondary sources whereas stars indicate primary sinks and diamonds indicate secondary sinks.

The proteins homologous to BAT5 in Arabidopsis are obviously not important to transport GSL biosynthesis intermediates, as the triple bat 3 bat 4 bat 5 mutant was not more affected in GSL biosynthesis than the single bat5 mutant was. Once keto-acids are imported into chloroplasts by BAT5, they enter several cycles of reactions including: condensation with acetyl-CoA catalyzed by a MAM, isomerization by an IPMI, and oxidative decarboxylation by an IPMDH Gigolashvili et al., (2009); Knill et al., (2009); Sawada et al., (2009 a, b). Elongated ketoacid can be again aminated by BCATs to yield the side-chain elongated Met (homoMet), which either is channeled into GSL biosynthesis or can proceed through another round of chain elongations to yield dihomoMet, trihomoMet, etc. For detailed review see Grubb and Abel, (2006); Sonderby et al., (2010). The obtained 2- keto-acids with longer carbon chain, or derived amino acid, need to be exported from the chloroplast into the cytoplasm to be incorporated into the GSL core biosynthetic pathway. However, the identity of this transporter, which is involved in the export of amino acid, is unknown. It was also not possible to analyze whether ketoacids or amino acids are preferentially exported from the chloroplasts. Theoretically, the export of both types' substrates is possible from chloroplasts as the transamination (and deamination reactions) of keto both chloroplastidic BCAT3 Knill et al., (2008) and cytoplasmic BCAT4 Schuster et al., (2006), could catalyze acids. It is rational to suppose that the BAT5, which imports the 2-keto-acid MTOB into the 
chloroplasts, also exports the chain-elongated 2-keto-acids back into the cytoplasm. However, attempts to measure MTOB and homomethionine transport activity of recombinant BAT5 in vitro were not successful till now, as the direct measurements of transport of these substrates in artificial membranes is problematic due to higher hydrophobicity of substrates leading to unspecific membrane binding and permeation, which override the specific transport events Gigolashvili et al., (2009). New technologies and approaches are needed to find out whether BAT5 is a dual transporter and if not, to identify the exporter of the elongated Met.

\section{Catabolism, Storage and Transport of Methionine}

SAMS directs about $80 \%$ of the metabolic flux of methionine to SAM, which is used by SAMdependent methyltransferases (MTs) to methylate nucleic acids, proteins and cell wall components like lipids, lignins and pectins Shen et al. (2002); Yang et al. (2006). SAM is also substrate for nicotianamine synthase (EC 2.5.1.43), SAM-decarboxylase (EC 4.1.1.50) and 1-aminocyclopropane- 1-carboxylate synthase (EC 4.4.1.14) to produce nicotianamine, polyamines and ethylene, respectively Hesse et al. (2004). These results demonstrate that SAM is (1) the second most frequently used cofactor in nature, after ATP Cantoni (1975); Lu (2000) and (2) mandatory for proper development of plants by influencing metal homeostasis and hormone function Burstenbinder et al. (2007); Klatte et al. (2009). Fig. (17). Interestingly, SAMS1 activity can be efficiently inhibited by S-nitrosylation by the natural NO donor S-nitrosoglutathione, which may mediate cross talk between NO and ethylene-signaling pathways in plants Lindermayr et al. (2005) Two cytosolic cycles regenerate methionine and SAM. First, the transfer of the methyl group from SAM generates S-adenosylhomocysteine (SAH), a potent inhibitor of MTs. To mitigate the toxic effects of SAH on MT activity and to recycle methionine, SAH is removed by SAH hydrolase (EC 3.3.1.1) in a reaction generating adenosine and homocysteine Hesse et al. (2004).
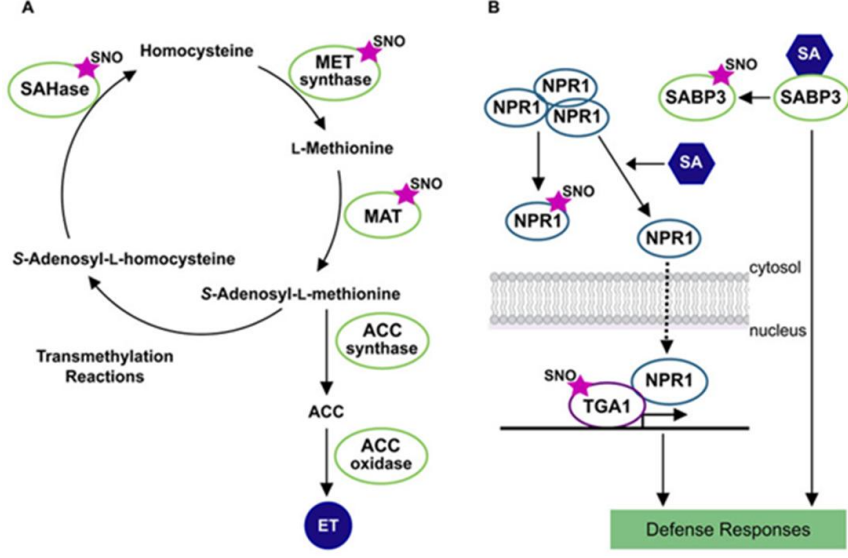

Fig. 17: Illustrate S-nitrosylation of target proteins in ethylene biosynthesis and salicylic acid network. The figure shows a schematic representation of methylmethionine cycle in the ethylene (ET) synthesis (A) and salicylic acid (SA) signaling networks (B). Protein S-nitrosothiols are represented by an SNO mark. References to physiological processes regulated by hormones, and subcellular localizations in the cell are also indicated. SAHase, adenosylhomocysteinase; MET synthase, cobalamin-independent methionine synthase; MAT, methionine adenosyltransferase; ACC, aminocyclopropane-1-carboxylic acid; SABP3, salicylic acid binding protein 3; NPR1, non-expresser of pathogenesis-related gene1 protein; TGA1, transcription factor TGA1 after Ramiro et al (2013)

The latter can be used as building block for synthesis of methionine MS as described above. Second, recycling of methionine at high rates of ethylene production is achieved by the Yang- or Metcycle Adams and Yang (1977) Fig.(18), which converts methylthioadenosine, the byproduct of SAM dependent ethylene formation in four steps to methionine. Recently, analysis of methylthioribose kinase (EC 2.7.1.100) revealed the significance of methionine recycling under sulfur limiting conditions in Arabidopsis Burstenbinder et al. (2007). Besides consumption of methionine in form of SAM, methionine can be catabolized in the cytosol by the activity of methionine g-lyase (MGL, EC 4.4.1.11), which produces ammonia, 2-oxobutanoate and methanthiol Rebeille et al. (2006). Methanthiol can be incorporated into cysteine, while 2-oxobutanoate can serve as a precursor for isoleucine synthesis Rebeille et al. (2006); Goyer et al. (2007). 


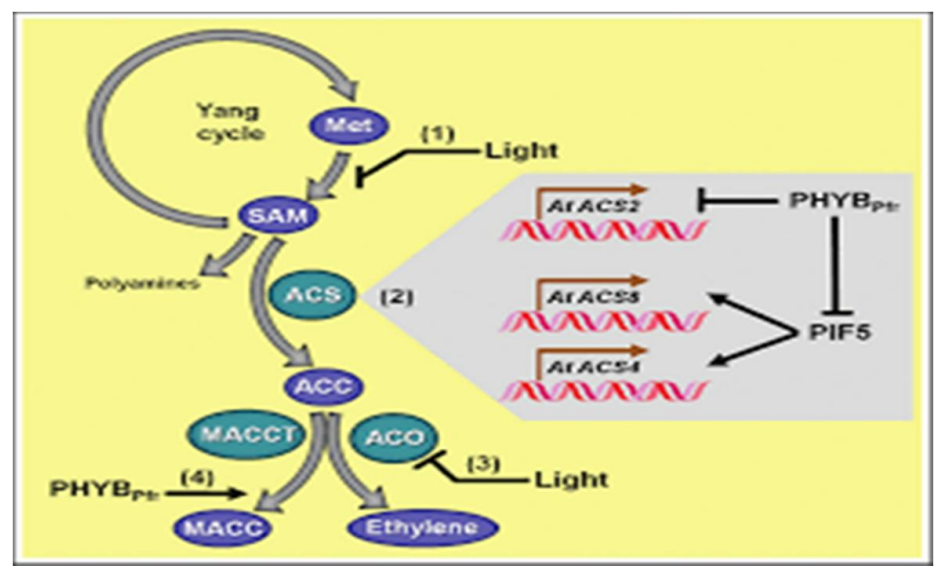

Fig. 18: Schematic representation of light-evoked impacts on ethylene biosynthetic pathway. (1) Light irradiation negatively affects the conversion of methionine (Met) to S-adenosyl methionine (SAM). (2) Light also affects the transcription of particular 1-aminocyclopropane 1-carboxylic acid (ACC) synthases (ACS), responsible for converting SAM into ACC. In Arabidopsis, whereas ACS2 gene transcription is negatively controlled by PHYBPfr, ACS8/4 gene transcription is stimulated by PIF5 and, accordingly, PIF5 protein stability is negatively influenced by active phytochrome B (PHYBPfr). (3) Light is also known to inhibit the transcription and activity of ACC oxidase (ACO), whose activity converts ACC into ethylene. (4) PHYBPfr also stimulates the conversion of ACC into the non-volatile ACC metabolite 1-malonyl aminocyclopropane-1-carboxylic acid (MACC) via MACC transferase (MACCT). Biosynthetic enzymes and metabolic substrates or products are represented with green and blue ovals, respectively. Light-dependent transcriptional control of particular ACS genes is highlighted by the gray area of the fig. After Maria et al (2014)

Under normal growth, condition a knockout of methionine g-lyase activity in Arabidopsis results in no visible phenotype, but a tenfold accumulation of methionine under sulfate-limiting conditions Goyer et al. (2007). Long distance transport of methionine is achieved via the phloem after conversion of methionine to S-methylmethionine (SMM, see Sect. 2.2), which is also assumed to function as temporary storage of methionine in leaves Bourgis et al. (1999). Methionine methyltransferase (EC 2.1.1.12) uses SAM as a methyl donor to form SMM from methionine and is unique in plants although it is nonessential Bourgis et al. (1999); Ranocha et al. (2000); Kocsis et al. (2005s).

\section{Conclusion}

Intense efforts have been devoted to describe the biochemical pathway of plant sulphur assimilation from sulphate. However, essential information on metabolic regulation of sulfur assimilation is still lacking, such as possible interactions between Sulfur assimilation, photosynthesis and photorespiration. Sulfur assimilation is stimulated by photo respiratory metabolism. Sulfurmetabolites represent a small portion of plant metabolites, the pathways of their synthesis are complex and their function and homeostasis require a large number of intra and intercellular transport steps. Transporters have been characterized, with some of the main gaps, many other transporters still await discovery, above all the transporter responsible for a major flux of sulfate in the cells. Sulfur-metabolites represent a small portion of plant metabolites, the pathways of their synthesis are complex and their function and homeostasis require a large number of intra and intercellular transport steps. New tools and approaches, e.g., 34S labeling, exploitation of natural variation, and mathematical modeling will be necessary to obtain the comprehensive list of S-metabolite transporters and to understand the role they play in controlling sulfur metabolism and homeostasis in plants. The increased use of these approaches in sulfur research makes hope for a rapid progress in dissection of the network of transporters in sulfur metabolism.

\section{References}

Aarabi F., K. Miyuki, T. Takayuki, K. Tomokazu, G. Tamara, T. Makiko, S. Yoko, W. Mutsumi, N. Hideo, R.F. Alisdair, S. Kazuki, T. Hideki, H. Hans-Michael, H. Rainer, M.-N. Akiko, 2016. 
Sulfur deficiency-induced repressor proteins optimize glucosinolate biosynthesis in plants. Sci. Adv. 2: e1601087

Abola, A.P., M.G. Willits, R.C. Wang, and S.R. Long, 1999. Reduction of adenosine-5 -phosphosulfate instead of 3 -phosphoadenosine- 5 -phosphosulfate in cysteine biosynthesis by Rhizobium meliloti and other members of the family Rhizobiaceae. J. Bacteriol. 181, 5280-5287.

Adams, D.O., S.F. Yang, 1977.Methionine metabolism in apple tissue: implication of Sadenosylmethionine as an intermediate in the conversion of methionine to ethylene. Plant Physiol 60:892-896.

Ågren, G. I., and M. Weih, 2012. Plant stoichiometry at different scales: element concentration patterns reflect environment more than genotype. New Phytol. 194: 944-952.

Amir R, Y. Hacham, G. Galili, 2002. Cystathionine g-synthase and threonine synthase operate in concert to regulate carbon flow towards methionine in plants. Trends Plant Sci. 7:153-156.

Aubry, S., R.D. Smith-Unna, C.M. Boursnell, S. Kopriva, and J. M. Hibberd, 2014.Transcript residency on ribosomes reveals a key role for the Arabidopsis thaliana bundle sheath in sulfur and glucosinolate metabolism. Plant J. 78, 659- 673 doi: 10.1111/tpj.12502

Badiani, M., A. D'annibale, A. Paolacci, F. Miglietta, and A. Raschi, 1993. The antioxidant status of soybean (Glycine max.leaves grown under natural $\mathrm{CO} 2$ enrichment in the field. Funct. Plant Biol. 20: $275-284$

Bak, S., and R. Feyereisen, 2001.The involvement of two P450 enzymes, CYP83B1 and CYP83A1, in auxin homeostasis and glucosinolate biosynthesis. Plant Physiol. 127: 108-118. doi: 10.1104/pp.127.1.108.

Baxter, I., B. Muthukumar, H.C. Park, P. Buchner, B. Lahner, J. Danku, K. Zhao, J. Lee, M.J. Hawkesford, M.L. Guerinot, and D.E. Salt, 2008. Variation in molybdenum content across broadly distributed populations of Arabidopsis thaliana is controlled by a mitochondrial molybdenum transporter (MOT1.PLoS Genet 4:e1000004.

Bell, C.S., W.J. Cram, and D.T. Clarkson, 1994. Compartmental analysis of 35 SO4 2 exchange kinetics in roots and leaves of a tropical legume Macroptilium atropurpureum cv. Siratro. J. Exp. Bot. 45:879-886.

Bennett R. N and R. M. Wallsgrove, 1994. Transley Review No.72. Secondary metabolites in plant defence mechanisms, New Phytologist, 127(4): 617-633,

Birke, H., S. J. Muller, M. Rother, A. D. Zimmer, S. N. Hoernstein, D. Wesenberg, et al., 2012. The relevance of compartmentation for cysteine synthesis in phototrophic organisms. Protoplasma 249(Suppl. 2), S147-S155. doi: 10.1007/s00709-012-0411-9.

Blake-Kalff, M.M., K.R. Harrison, M.J. Hawkesford, F.J. Zhao, and S.P. McGrath, 1998. Distribution of sulfur within oilseed rape leaves in response to sulfur deficiency during vegetative growth. Plant Physiol., 118:1337-1344.

Bloom, A. J., M. Burger, J.S.R. Asensio, and A.B. Cousins, 2010. Carbon dioxide Enrichment inhibits nitrate assimilation in wheat and Arabidopsis. Science, 328: 899-903.

Bochenek, M., G. J. Etherington, A. Koprivova, S. T. Mugford, T. G. Bell, G. Malin, et al., 2013. Transcriptome analysis of the sulfate deficiency response in the marine microalga Emiliania huxleyi. New Phytol. 199, 650-662. doi:

Bogs, J., A. Bourbouloux, O. Cagnac, A. Wachter, T. Rausch, and S. Delrot, 2003. Functional characterization and expression analysis of a glutathione transporter, BjGT1, from Brassica juncea: evidence for regulation by heavy metal exposure. Plant Cell Environ. 26: 1703-1711. doi: 10.1046/j.1365-3040.2003.01088.x.

Bourbouloux, A., P. Shahi, A. Chakladar, S. Delrot, and A. K. Bachhawat, 2000. Hgt1p, a high affinity glutathione transporter from the yeast Saccharomyces cerevisiae. J. Biol. Chem. 275, 1325913265. doi: $10.1074 /$ jbc.275.18.13259.

Bourgis, F., S. Roje, M. L. Nuccio, D. B. Fisher, M. C. Tarczynski, C. Li, et al., 1999. Smethylmethionine plays a major role in phloem sulfur transport and is synthesized by a novel type of methyltransferase. Plant Cell, 11: 1485-1498. doi: 10.1105/tpc.11.8.1485.

Bouvier, F., N. Linka, J. C. Isner, J. Mutterer, A. P. Weber, and B. Camara, 2006. Arabidopsis SAMT1 defines a plastid transporter regulating plastid biogenesis and plant development. Plant Cell 18: 3088-3105. doi: 10.1105/tpc.105.040741. 
Brunold, C., 1993. Regulatory interactions between sulfate and nitrate assimilation, Sulfur Nutrition and Sulfur Assimilation in Higher Plants, eds L. J. De Kok, I. Stulen, H. Rennenberg, C. Brunold, and W. E. Rauser (Hague: SPB Academic Publishing), 61-75.

Brunold, C., 2003. Effect of glucose on assimilatory sulphate reduction in Arabidopsis thaliana roots. J. Exp. Bot. 54: 1701-1709.

Buchner, P., H. Takahashi, and M.J. Hawkesford, 2004a. Plant sulphate transporters: co-ordination of uptake, intracellular and long-distance transport. J. Exp. Bot., 55:1765-1773.

Buchner, P., S. Parmar, A. Kriegel, M. Carpentier, and M. J. Hawkesford, 2010.The sulfate transporter family in wheat: tissue-specific gene expression in relation to nutrition. Mol. Plant 3: 374-389. doi: $10.1093 / \mathrm{mp} / \mathrm{ssp} 119$.

Buchner, P., C. E. E. Stuiver, S. Westerman, M. Wirtz, R. Hell, M. J. Hawkesford, et al., 2004a. Regulation of sulfate uptake and expression of sulfate transporter genes in Brassica oleracea as affected by atmospheric H2S and pedospheric sulfate nutrition. Plant Physiol. 136: 3396-3408. doi: $10.1104 /$ pp.104.046441.

Buchner, P., H. Takahashi, and M. J. Hawkesford, 2004b. Plant sulphate transporters: co-ordination of uptake, intracellular and long-distance transport. J. Exp. Bot. 55: 1765-1773. doi: $10.1093 / \mathrm{jxb} / \mathrm{erh} 206$.

Burgener, M., M. Suter, S. Jones, and C. Brunold, 1998. Cyst(e)ine is the transport metabolite of assimilated sulfur from bundle-sheath to mesophyll cells in maize leaves. Plant Physiol 116:1315-1322

Burstenbinder, K., G. Rzewuski, M. Wirtz, R. Hell, and M. Sauter, 2007.The role of methionine recycling for ethylene synthesis in Arabidopsis. Plant J., 49:238-249.

Cagnac, O., A. Bourbouloux, D. Chakrabarty, M.-Y. Zhang, and S. Delrot, 2004. AtOPT6 transports glutathione derivatives and is induced by primisulfuron. Plant Physiol 135:1378-1387

Cantoni, G.L., 1975.Biological methylation: selected aspects. Annu Rev Biochem 44:435-451 Chen S, Petersen BL, Olsen CE, Schulz A, Halkier BA, 2001. Long-distance phloem transport of glucosinolates in Arabidopsis. Plant Physiol., 127:194-201.

Cao, M. J., Z. Wang, M. Wirtz, R. Hell, D. J. Oliver, and C. B. Xiang, 2013. SULTR3;1 is a chloroplastlocalized sulfate transporter in Arabidopsis thaliana. Plant J. 73, 607-616. doi: 10.1111/tpj.12059

Casieri, L., K. Gallardo, and D. Wipf, 2012.Transcriptional response of Medicago truncatula sulphate transporters to arbuscular mycorrhizal symbiosis with and without sulphur stress. Planta 235: 1431-1447. doi: 10.1007/s00425-012-1645-7.

Chan, K. X., M. Wirtz, S. Y. Phua, G. M. Estavillo, and B. J. Pogson, 2013. Balancing metabolites in drought: the sulfur assimilation Conundrum. Trends Plant Sci.18: 18-29.

Chen, S., E. Glawischnig, K. Jørgensen, P. Naur, B. Jørgensen, C. E. Olsen, et al., 2003. CYP79F1 and CYP79F2 have distinct functions in the biosynthesis of aliphatic glucosinolates in Arabidopsis. Plant J. 33: 923-937. doi: 10.1046/j.1365- 313X.2003.01679.x.

Collakova, E., 2008. Arabidopsis 10-formyl tetrahydrofolate deformylases are Essential for photorespiration. Plant Cell 20: 1818-1832.

Dahl, C., R. Hell, T. Leustek, and D. Knaff, 2008. Introduction to sulfur metabolism in phototrophic organisms. In: Hell R, Dahl C, Leustek T (eds).Sulfur metabolism in phototrophic organisms. Springer, Dordrecht, The Netherlands, 1-16.

Davies, J. P., F. H. Yildiz, and A. Grossman, 1996. Sac1, a putative regulator that is critical for survival of Chlamydomonas reinhardtii during sulfur deprivation. EMBO J. 15, 2150-2159.

De Kraker, J.-W., and J. Gershenzon, 2011. From amino acid to glucosinolate biosynthesis: protein sequence changes in the evolution of methylthioalkylmalate synthase in Arabidopsis. Plant Cell, 23: 38-53. doi: 10.1105/tpc.110.079269.

Douce, R., J. Bourguignon, M. Neuburger, and F. Rébeillé, 2001. The glycine Decarboxylase system: a fascinating complex. Trends Plant Sci., 6: 167-176.

Douglas Grubb, C., B. J. Zipp, J. Kopycki, M. Schubert, M. Quint, E. K. Lim, et al., 2014. Comparative analysis of Arabidopsis UGT74 glucosyltransferases reveals a special role of UGT74C1 in glucosinolate biosynthesis. Plant J. 79: 92-105. doi: 10.1111/tpj.12541.

Dubousset, L., M. Abdallah, A.S. Desfeux, P. Etienne, F. Meuriot, M.J. Hawkesford, J. Gombert, R. Segura, M.P. Bataille, S. Reze, J. Bonnefoy, A.F. Ameline, A. Ourry, F. Le Dily, and J.C. Avice, 2009. Remobilization of leaf $S$ compounds and senescence in response to restricted sulphate 
supply during the vegetative stage of oilseed rape are affected by mineral N availability. J. Exp. Bot. 60:3239-3253.

Eichel, J., J. C. Gonzalez, M. Hotze, R. G. Matthews, and J. Schroder, 1995. Vitamin-B12-independent methionine synthase from a higher plant (Catharanthus roseus.) Molecular characterization, regulation, heterologous expression, and enzyme properties. Eur. J. Biochem. 230: 1053-1058. doi: 10.1111/j.1432-1033.1995.tb20655.x.

Falkowski, P.G., 2006. Evolution: tracing oxygen's imprint on earth's metabolic evolution. Science 311:1724-1725.

Ferreira, R.M., and A.R. Teixeira, 1992. Sulfur starvation in Lemna leads to degradation of ribulose bisphosphate carboxylase without plant death. J. Biol. Chem., 267:7253-7257.

Ferretti, M., Destro, T., S.C.E. Tosatto, L. La Rocca, N. Rascio, and A. Masi, 2009. g-glutamyl transferase in the cell wall participates in extracellular glutathione salvage from the root apoplast. New Phytol., 181:115-126

Gigolashvili, T., R. Yatusevich, I. Rollwitz, M. Humphry, J. Gershenzon, and U.-I. Fluegge, 2009. The plastidic bile acid transporter 5 is required for the biosynthesis of methionine-derived glucosinolates in Arabidopsis thaliana. Plant Cell 21: 1813-1829. doi: 10.1105/tpc.109.066399

Gilbert, S. M.,. D. T. Clarkson, M. Cambridge, H. Lambers, and M. J. Hawkesford, 1997. S042deprivation has an early effect on the content of ribulose -1,5-bisphosphat carboxylase/oxygenase and photosynthesis in young leaves of wheat, Plant Physiology, 115(3): 1231-1239,

Goyer, A., E. Collakova, Y. Shachar-Hill, and A.D. Hanson, 2007. Functional characterization of a methionine g-lyase in Arabidopsis and its implication in an alternative to the reverse transsulfuration pathway. Plant Cell Physiol., 48:232-242.

Gromes, R., M. Hothorn, E.D. Lenherr, V. Rybin, K. Scheffzek, and T. Rausch, 2008. The redox switch of g-glutamylcysteine ligase via a reversible monomer-dimer transition is a mechanism unique to plants. Planta, 180:262-271.

Grubb, C. D., and S. Abel, 2006. Glucosinolate metabolism and its control. Trends Plant Sci. 11: 89100. doi: 10.1016/j.tplants.2005.12.006.

Hall, J. L., 2002.Cellular mechanisms for heavy metal detoxification and tolerance. J. Exp. Bot. 53: 111. doi: $10.1093 /$ jexbot/53.366.1.

Hansen, C. H., U. Wittstock, C. E. Olsen, A. J. Hick, J. A. Pickett, and B. A. Halkier, 2001. Cytochrome P450 CYP79F1 from Arabidopsis catalyzes the conversion of dihomomethionine and trihomomethionine to the corresponding aldoximes in the biosynthesis of aliphatic glucosinolates. J. Biol. Chem. 276: 11078-11085. doi: 10.1074/jbc.M010123200.

Hawkesford, M.J., and L.J. De Kok, 2006. Managing sulphur metabolism in plants. Plant Cell Environ 29:382-395.

Heeg, C., C. Kruse, R. Jost, M. Gutensohn, T. Ruppert, M. Wirtz, and R. Hell, 2008. Analysis of the Arabidopsis O-acetylserine(thiol)lyase gene family demonstrates compartment -specific differences in the regulation of cysteine synthesis. Plant Cell, 20:168-185.

Hell, R., H. Hillebrand, 2001. Plant concepts for mineral acquisition and allocation. Curr Opin Biotechnol 12:161-168.

Hemm, M. R., M. O. Ruegger, and C. Chapple, 2003. The Arabidopsis ref2 mutant is defective in the gene encoding CYP83A1 and shows both phenylpropanoid and glucosinolate phenotypes. Plant Cell 15: 179-194. doi: 10.1105/tpc.006544.

Herschbach, C., and H. Rennenberg, 1995. Long-distance transport of S-35- sulphur in 3-year-old beech trees (Fagus sylvatica.Physiol. Plant. 95: 379-386. doi: 10.1111/j.1399-3054.1995.tb00852.x.

Hesse, H., O. Kreft, S. Maimann, M. Zeh, R. Hoefgen, 2004. Current understanding of the regulation of methionine biosynthesis in plants. J. Exp. Bot., 55:1799-1808.

Hesse, H., O. Kreft, S. Maimann, M. Zeh, and R. Hoefgen, 2004. Current understanding of the regulation of methionine biosynthesis in plants. J. Exp. Bot. 55: 1799-1808. doi: 10.1093/jxb/erh139.

Hirai, M., T. Fujiwara, M. Awazuhara, T. Kimura, M. Noji, and K. Saito, 2003. Global expression profiling of sulfur-starved Arabidopsis by DNA macroarray reveals the role of O-acetyl-L-serine as a general regulator of gene expression in response to sulfur nutrition. Plant J., 33:651-663.

Hirai, M. Y., T. Fujiwara, M. Awazuhara, T. Kimura, M. Noji, and K. Saito, 2003. Global expression profiling of sulfur-starved Arabidopsis by DNA macroarray reveals the role of O-acetyl-1-serine 
as a general regulator of gene expression in response to sulfur nutrition. Plant J. 33: 651-663. doi: 10.1046/j.1365-313X. 2003.01658.x.

Hirai, M. Y., M. Klein, Y. Fujikawa, M. Yano, D. B. Goodenowe, Y. Yamazaki, et al., 2005. Elucidation of gene-to-gene and metabolite-to-gene networks in Arabidopsis by integration of metabolomics and transcriptomics. J. Biol. Chem. 280: 25590-25595. doi: 10.1074/jbc.M502332200.

Högy, P., and A. Fangmeier, 2008. Effects of elevated atmospheric CO2 on grain quality of wheat. J. Cereal Sci. 48: 580-591.

Högy, P., 2009. Effects of elevated CO2 on grain yield and quality of wheat: results from a 3-year freeair CO2 enrichment experiment. Plant Biol. 11(s1): 60-69 .

Hopkins, L., S. Parmar, A. Blaszczyk, H. Hesse, R. Hoefgen, and M.J. Hawkesford, 2005. Oacetylserine and the regulation of expression of genes encoding components for sulfate uptake and assimilation in potato. Plant Physiol., 138:433-440.

Hothorn, M., A. Wachter, R. Gromes, T. Stuwe, T. Rausch, and K. Scheffzek, 2006. Structural basis for the redox control of plant glutamate cysteine ligase. J. Biol. Chem., 281:27557-27565.

Howarth, J.R., S. Parmar, J. Jones, C.E. Shepherd, D.I. Corol, A.M. Galster, N.D. Hawkins, S.J. Miller, J.M. Baker, P.J. Verrier, J.L. Ward, M.H. Beale, P.B. Barraclough, M.J. Hawkes ford, 2008. Coordinated expression of amino acid metabolism in response to $\mathrm{N}$ and $\mathrm{S}$ deficiency during wheat grain filling. J. Exp. Bot., 59:3675-3689.

Hubberten, H. M., S. Klie, C. Caldana, T. Degenkolbe, L. Willmitzer, and R. Hoefgen, 2012 b. Additional role of O-acetylserine as a sulfur statusindependent regulator during plant growth. Plant J. 70: 666-677. Doi: 10.1111/J.1365-313X.2012.04905.x.

Hubberten, H. M., S. Klie, C. Caldana, T. Degenkolbe, L. Willmitzer, and R. Hoefgen, 2012. Additional role of $\mathrm{O}$-acetylserine as a sulfur status-independent regulator during plant growth. Plant J. 70: 666-677. doi: 10.1111/j.1365- 313X.2012.04905.x.

Jamal, A., I.S. Fazli, S. Ahmad, M.Z. Abdin, 2006a. Interactive effect of nitrogen and sulphur on yield and quality of groundnut (Arachis hypogea L.) Korean J. Crop. Sci., 51(6): 519-522.

Jamal, A., I.S. Fazli, S. Ahmad, M.Z. Abdin, and S.J. Yun, 2005. Effect of sulphur and nitrogen application on growth characteristics, seed and oil yield of soybean cultivars. Korean J. Crop. Sci., 50(5): 340-345.

Jamal, A., I.S. Fazli, S. Ahmad, M.Z. Abdin, and S.J. Yun, 2006c. Effectof nitrogen and sulphur application on nitrate reductase and ATP- sulphurylase activities in Soybean. Korean, J. Crop. Sci., 51(4): 298-302.

Jamal, A., I.S. Fazli, S. Ahmad, K.T. Kim, D.G. Oh, and M. Abdin, 2006b. Effect of sulfur on nitrate reductase and ATPsulfurylase activities in groundnut (Arachis hypogea L.) J. Plant Biol., 49(6): 513-517.

Jamal, A., K. Ko, H.S. Kim, Y.K. Cho, H. Joung, and K. Ko, 2009. Role of genetic factors and environmental conditions in recombinant protein production for plant molecular biofarming. Biotech. Advan., 27: 914-923.

Jamal, A., Y.S. Moon, and M.Z. Abdin, 2010. Enzyme activity assessment of peanut (Arachis hypogea.under slow-release sulphur fertilization. Aust J Crop Sci 4(3):169-174

Jaspers, P., and J. Kangasj, 2010. arvi Reactive oxygen species in abiotic stress signaling, Physiologia Plantarum, 138(4): 405-413,.

Jez, J.M., R.E. Cahoon, and S. Chen, 2004. Arabidopsis thaliana glutamate-cysteine ligase: functional properties, kinetic mechanism, and regulation of activity. J. Biol. Chem., 279:33463-33470.

Jia, Y., 2010. Effects of elevated CO2 on growth, photosynthesis, elemental composition, antioxidant level, and phytochelatin concentration in Lolium mutiforum and Lolium perenne under Cd stress. J. Hazard. Mater. 180: 384-394.

Kataoka, T., N. Hayashi, T. Yamaya, and H. Takahashi, 2004a. Root-to-shoot transport of sulfate in Arabidopsis. Evidence for the role of SULTR3;5 as a component of low-affinity sulfate transport system in the root vasculature. Plant Physiol., 136:4198-4204.

Kataoka, T., A. Watanabe-Takahashi, N. Hayashi, M. Ohnishi, T. Mimura, P. Buchner, M.J. Hawkesford, T. Yamaya, and H. Takahashi, 2004b. Vacuolar sulfate transporters are essential determinants controlling internal distribution of sulfate in Arabidopsis. Plant Cell, 16:2693-2704. 
Kaur, J., and A. K. Bachhawat, 2007. Yct1p, a novel, high-affinity, cysteine-specific transporter from the yeast Saccharomyces cerevisiae. Genetics 176: 877-890. doi: 10.1534/genetics.107.070342.

Kawashima, C. G., C. A. Matthewman, S. Q. Huang, B. R. Lee, N. Yoshimoto, A. Koprivova, et al., 2011. Interplay of SLIM1 and miR395 in the regulation of sulfate assimilation in Arabidopsis. Plant J. 66, 863-876. doi: 10.1111/j.1365-313X.2011.04547.x.

Kawashima, C. G., N. Yoshimoto, A. Maruyama-Nakashita, Y. N. Tsuchiya, K. Saito, H. Takahashi, et al., 2009. Sulphur starvation induces the expression of microRNA-395 and one of its target genes but in different cell types. Plant J. 57: 313-321. doi: 10.1111/j.1365-313X.2008.03690.x.

Klatte, M., M. Schuler, M. Wirtz, C. Fink-Straube, R. Hell, and P. Bauer, 2009. The analysis of Arabidopsis nicotianamine synthase mutants reveals functions for nicotianamine in seed iron loading and iron deficiency responses. Plant Physiol., 150:257-271.

Knill, T., M. Reichelt, C. Paetz, J. Gershenzon, and S. Binder, 2009. Arabidopsis thaliana encodes a bacterial-type heterodimeric isopropylmalate isomerase involved in both Leu biosynthesis and the Met chain elongation pathway of glucosinolate formation. Plant Mol. Biol. 71: 227-239. doi: 10.1007/s11103-009- 9519-5.

Knill, T., J. Schuster, M. Reichelt, J. Gershenzon, and S. Binder, 2008. Arabidopsis branched-chain aminotransferase 3 functions in both amino acid and glucosinolate biosynthesis. Plant Physiol. 146: 1028-1039. doi: 10.1104/pp.107.111609.

Kocsis, M.G, P. Ranocha, D.A. Gage, E.S. Simon, D. Rhodes, G.J. Peel, S. Mellema, K. Sito, M. Awazu hara, G. Li, R.B. Meeley, M.C. Tarczynski, C. Wagner, and A.D. Hanson, 2003. Insertional in activation of the methionine-s methyltransferase gene elimination the smethymethionine cycle and increase the methylation ratio. Plant Physiol. 131: 1808-1815.

Kopriva, S., and A. Koprivova, 2005. Sulfate assimilation and glutathione synthesis in C-4 plants. Photosynth. Res. 86: 363-372. doi: 10.1007/s11120-005-3482-z.

Kopriva, S., S. G. Mugford, C. Matthewman, and A. Koprivova, 2009. Plant sulfate assimilation genes: redundancy versus specialization. Plant Cell Rep. 28: 1769-1780. doi: 10.1007/s00299-0090793-0.

Kopriva, S., 2002. Interaction of sulfate assimilation with carbon and nitrogen metabolism in Lemna minor. Plant Physiol. 130: 1406-1413.

Koprivova, A., A.J. Meyer, G. Schween, C. Herschbach, R. Reski, and S. Kopriva, 2002. Functional knockout of the adenosine 50-phosphosulfate reductase gene in Physcomitrella patens revives an old route of sulfate assimilation. J. Biol. Chem., 277:32195-32201.

Koprivova, A., S. T. Mugford, and S. Kopriva, 2010. Arabidopsis root growth dependence on glutathione is linked to auxin transport. Plant Cell Rep. 29: 1157- 1167. doi: 10.1007/s00299010-0902-0.

Koprivova, A., M. Suter, R. Op den Camp, C. Brunold, and S. Kopriva, 2000. Regulation of sulfate assimilation by nitrogen in Arabidopsis. Plant Physiol. 122: 737-746. doi: 10.1104/pp.122.3.737

Koralewska, A., P. Buchner, C. E. E. Stuiver, F. S. Posthumus, S. Kopriva, M. J. Hawkesford, et al., 2009. Expression and activity of sulfate transporters and APS reductase in curly kale in response to sulfate deprivation and re-supply. J. Plant Physiol. 166: 168-179. doi: 10.1016/j.jplph.2008.03.005.

Kruse, C., R. Jost, M. Lipschis, B. Kopp, M. Hartmann, and R. Hell, 2007. Sulfur-enhanced defence: effects of sulfur metabolism, nitrogen supply, and pathogen lifestyle. Plant Biol., 9:608-619

Kulich, I., T. Pe`cenková, J. Sekereš, O. Smetana, M. Fendrych, I. Foissner, et al., 2013. Arabidopsis exocyst subcomplex containing subunit EXO70B1 is involved in autophagy-related transport to the vacuole. Traffic 14: 1155-1165. doi: 10.1111/tra.12101.

Kutz, A., A. Muller, P. Hennig, W.M. Kaiser, M. Piotrowski, and E.W. Weiler, 2002. A role for nitrilase 3 in the regulation of root morphology in sulphur-starving Arabidopsis thaliana. Plant J. 30:95106

Kazuki, S., 2004. Sulfur Assimilatory Metabolism. The Long and Smelling Road. Plant Physiology, 136: $2443-2450$.

Lamb, J. R., S. Tugendreich, and P. Hieter, 1995. Tetratrico peptide repeat interactions: To Tpr or not to Tpr. Trends Biochem. Sci., 20: 257-259. 
Lee, B. R., A. Koprivova, and S. Kopriva, 2011. The key enzyme of sulfate assimilation, adenosine 5 phosphosulfate reductase, is regulated by HY5 in Arabidopsis. Plant J. 67: 1042-1054. doi: 10.1111/j.1365-313X.2011.04656.x.

Lee, C. P., M. Wirtz, and R. Hell, 2014. Evidence for several cysteine transport mechanisms in the mitochondrial membranes of Arabidopsis thaliana. Plant Cell Physiol. 55: 64-73. doi: $10.1093 / \mathrm{pcp} / \mathrm{pct} 155$.

Leustek, T., and K. Saito., 1999. Sulfate transport and assimilation in plants, Plant Physiology, 120 (3): 637-643.

Lewandowska, M., and A. Sirko, 2008. Recent advances in understanding plant response to sulfur deficiency stress. Acta Biochim Pol 55:457-471.

Liang, G., F. Yang, and D. Yu, 2010. MicroRNA395 mediates regulation of sulfate accumulation and allocation in Arabidopsis thaliana. Plant J. 62: 1046-1057.

Lindermayr, C., G. Saalbach, and J. Durner, 2005. Proteomic identification of S-nitrosylated proteins in Arabidopsis. Plant Physiol., 137:921-930.

Lu, S.C., 2000. S-Adenosylmethionine. Int J Biochem Cell Biol 32:391-395.

Lunn, J.E., M. Droux, J. Martin, and R. Douce, 1990. Localization of ATP-sulfurylase and Oacetylserine (thiol)lyase in spinach leaves. Plant Physiol., 94:1345-1352.

Marcell, N., H. Hans-Michael, W. Mutsumi, H. Robert, A. S. Mark and H. Rainer, 2018. Sulfite Reductase Co-suppression in Tobacco Reveals Detoxification Mechanisms and Downstream, Responses, Comparable to Sulfate starvation, Plant Sci. 9 (1423): 1-18.

Maria, A.R., B. Ricardo, and F. Luciano, 2014. Sheddibg light on ethylene metabolism in higher plants .Plant Sc., 5:1-16.

Markovich, D., and P. S. Aronson, 2007. Specificity and regulation of renal sulfate transporters. Annu. Rev. Physiol. 69: 361-375. doi: 10.1146/annurev.physiol.69.040705.141319.

Martin, W., C. Rotte, M. Hoffmeister, U. Theissen, G. Gelius-Dietrich, S. Ahr, and K. Henze, 2003. Early cell evolution, eukaryotes, anoxia, sulfide, oxygen, fungi first (?), and a tree of genomes revisited. IUBMB Life, 55:193-204.

Maruyama-Nakashita, A., Y. Nakamura, T. Tohge, K. Saito, and H. Takahashi, 2006. Arabidopsis SLIM1 is a central transcriptional regulator of plant sulfur response and metabolism. Plant Cell 18:3235-3251.

Maruyama-Nakashita, A., Y. Nakamura, A. Watanabe-Takahashi, E. Inoue, T. Yamaya, and H. Takahashi, 2005. Identification of a novel cis-acting element conferring sulfur deficiency response in Arabidopsis roots. Plant J., 42:305-314.

Maruyama-Nakashita, A., E. Inoue, A. Watanabe-Takahashi, T. Yamaya, and H. Takahashi, 2003. Transcriptome profiling of sulfur-responsive genes in Arabidopsis reveals global effects of sulfur nutrition on multiple metabolic pathways. Plant Physiol. 132: 597-605. doi: 10.1104/pp.102.019802.

Maruyama-Nakashita, A., Y. Nakamura, A. Watanabe-Takahashi, T. Yamaya, and H. Takahashi, 2004a. Induction of SULTR1;1 sulfate transporter in Arabidopsis roots involves protein phosphorylation/dephosphorylation circuit for transcriptional regulation. Plant Cell Physiol. 45: 340-345. doi: 10.1093/pcp/pch029.

Maruyama-Nakashita, A., Y. Nakamura, T. Yamaya, and H. Takahashi, 2004b. A novel regulatory pathway of sulfate uptake in Arabidopsis roots: implication of CRE1/WOL/AHK4-mediated cytokinin-dependent regulation. Plant J. 38: 779- 789. doi: 10.1111/j.1365-313X.2004.02079.x.

Maughan, S. C., M. Pasternak, N. Cairns, G. Kiddle, T. Brach, R. Jarvis, et al., 2010. Plant homologs of the Plasmodium falciparum chloroquineresistance transporter, PfCRT, are required for glutathione homeostasis and stress responses. Proc. Natl. Acad. Sci. U.S.A. 107: 2331-2336. doi: 10.1073/pnas. 0913689107.

McBean, G. J., and J. Flynn, 2001. Molecular mechanisms of cystine transport. Biochem. Soc. Trans. 29, 717-722. doi: 10.1042/BST0290717 Meister, A., Tate, S. S., and Griffith, O. W., 1981.Gamma-glutamyl transpeptidase. Methods Enzymol. 77: 237-253. doi: 10.1016/S00766879(81)77032-0.

Meister, A., S.S. Tate, and O.W. Griffith, 1981. Gamma-glutamyl trans peptidase. Methods Enzymol. 77: 237-253.doi:10.1016/S0076-6879 (81)77032-0. 
Melis, A., and H.-C. Chen, 2005. Chloroplast sulfate transport in green algae - genes, proteins and effects. Photosynth Res., 86:299-307.

Meyer, A.J., and R. Hell, 2005. Glutathione homeostasis and redox-regulation by sulfhydryl groups. Photosynth Res., 86:435-457.

Meyer, Y., W. Siala, T. Bashandy, C. Riondet, F. Vignols, and J.P. Reichheld, 2008. Glutaredoxins and thioredoxins in plants. Biochim. Biophys. Acta., 1783:589-600.

Mikkelsen, M. D., P. Naur, and B. A. Halkier, 2004. Arabidopsis mutants in the C-S lyase of glucosinolate biosynthesis establish a critical role for indole3-acetaldoxime in auxin homeostasis. Plant J. 37, 770-777. doi: 10.1111/j.1365- 313X.2004.02002.x.

Miller, A.J., Q. Shen, and G. Xu, 2009. Freeways in the plant: transporters for N, P and S and their regulation. Curr. Opin. Plant Biol. 12:284-290.

Miranda, M., L. Borisjuk, A. Tewes, U. Heim, N. Sauer, U. Wobus, et al., 2001. Amino acid permeases in developing seeds of Vicia faba L.: expression precedes storage protein synthesis and is regulated by amino acid supply. Plant J. 28: 61-71. doi: 10.1046/j.1365-313X.2001.01129.x.

Mittler, R., 2006. Abiotic stress, the field environment and stresscombination, Trends in Plant Science, 11(1): 15-19.

Miller, I.M, P. E. Jensen, and A. Hansson, 2007. Oxidative modifications to cellular components in plants, Annual Review of Plant Biology, 58: 459-481.

Mugford, S.G., N. Yoshimoto, M. Reichelt, M. Wirtz, L. Hill, S.T. Mugford, Y. Nakazato, M. Noji, H. Takahashi, R. Kramell, T. Gigolashvili, U.I. Flugge, C. Wasternack, J. Gershenzon, R. Hell, K. Saito, and S. Kopriva, 2009. Disruption of adenosine-50-phosphosulfate kinase in Arabidopsis reduces levels of sulfated secondary metabolites. Plant Cell, 21:910-927.

Naur, P., B. L. Petersen, M. D. Mikkelsen, S. Bak, H. Rasmussen, C. E. Olsen, et al., 2003. CYP83A1 and CYP83B1, two nonredundant cytochrome P450 enzymes metabolizing oximes in the biosynthesis of glucosinolates in Arabidopsis. Plant Physiol. 133: 63-72. doi: 10.1104/pp.102.019240.

Naveen, C. J., J. M. Andreas, A. K. B. Sajid, Z. Zhi-Liang and L. Thomas, 2019. Arabidopsis cGlutamylcyclotransferase affects glutathione Content and root system architecture during sulfur starvation 221(3):1387-1397. Doi: 10.1111/nph.15466.

Ndamukong, I., A. Abdallat, C. Thurow, B. Fode, M. Zander, R. Weigel, and C. Gatz, 2007. SAinducible Arabidopsis glutaredoxin interacts with TGA factors and suppresses JA-responsive PDF1.2 transcription. Plant J. 50:128-139.

Nikiforova, V.J., J. Kopka, V. Tolstikov, O. Fiehn, L. Hopkins, M.J. Hawkesford, H. Hesse, and R. Hoefgen, 2005. Systems rebalancing of metabolism in response to sulfur deprivation, as revealed b metabolome analysis of Arabidopsis plants. Plant Physiol., 138:304-318.

Nikiforova, V. J., B. Gakiere, S. Kempa, M. Adamik, L. Willmitzer, H. Hesse, et al., 2004. Towards dissecting nutrient metabolism in plants: a systems biology case study on sulphur metabolism. J. Exp. Bot., 55: 1861-1870. doi: 10.1093/jxb/ erh177.

Nikiforova, V., J. Freitag, S. Kempa, M. Adamik, H. Hesse, and R. Hoefgen, 2003. Transcriptome analysis of sulfur depletion in Arabidopsis thaliana: interlacing of biosynthetic pathways provides response specificity. Plant J. 33: 633-650. doi: 10.1046/j.1365-313X.2003.01657.x.

Noctor, G., A. Mhamdi, S. Chaouch, Y. Han, J. Neukermans, B. Marquez-Garcia, et al., 2012. Glutathione in plants: an integrated overview. Plant Cell Environ. 35: 454-484. doi: 10.1111/j.1365-3040.2011.02400.x.

Ohana, E., D. Yang, N. Shcheynikov, and S. Muallem, 2009. Diverse transport modes by the solute carrier 26 family of anion transporters. J. Physiol. 587: 2179- 2185. doi: 10.1113/jphysiol.2008.164863 .

Ortiz, D. F., L. Kreppel, D. M. Speiser, G. Scheel, G. Mcdonald, and D. W. Ow, 1992. Heavy metal tolerance in the fission yeast requires an ATP-binding cassette-type vacuolar membrane transporter. EMBO J. 11: 3491-3499.

Palmieri, L., R. Arrigoni, E. Blanco, F. Carrari, M.I. Zanor, C. Studart-Guimaraes, A.R. Fernie, and F. Palmieri, 2006. Molecular identification of an Arabidopsis S-adenosylmethionine transporter. Analysis of organ distribution, bacterial expression, reconstitution into liposomes, and functional characterization. Plant Physiol., 142:855-865. 
Park, J., W. Y. Song, D. Ko, Y. Eom, T. H. Hansen, M. Schiller, et al., 2012. The phytochelatin transporters AtABCC1 and AtABCC2 mediate tolerance to cadmium and mercury. Plant J. 69: 278-288. doi: 10.1111/j.1365-313X.2011.04789.x.

Pasternak, M., B. Lim, M. Wirtz, R. Hell, C.S. Cobbett, and A.J. Meyer, 2008. Restricting glutathione biosynthesis to the cytosol is sufficient for normal plant development. Plant J. 53:999-1012.

Rachmilevitch, S., A. B. Cousins, and A. J. Bloom, 2004. Nitrate assimilation in plant Shoots depends on photorespiration. Proc. Natl. Acad. Sci. USA, 101:11506-11510.

Ramiro Paris,Maria J.,Iglesias,Maria,C.Terrile and Claudia Casalongue, 2013. Functions of Snitrosylation in plant hormones networks. Pant Sc. 4 :1-6.

Rausch, T., A. Wachter, 2005. Sulfur metabolism: a versatile platform for launching defence operations. Trends Plant Sci 10:503-509.

Ravanel, S., M.A. Block, P. Rippert, S. Jabrin, G. Curien, F. Rebeille, and R. Douce, 2004. Methionine metabolism in plants: chloroplasts are autonomous for de novo methionine synthesis and can import S-adenosylmethionine from the cytosol. J. Biol. Chem. 279:22548-22557.

Rebeille, F., S. Jabrin, R. Bligny, K. Loizeau, B. Gambonnet, V. Van Wilder, R. Douce, and S. Ravanel, 2006. Methionine catabolism in Arabidopsis cells is initiated by a g-cleavage process and leads to S-methylcysteine and isoleucine syntheses. PNAS 103:15687-15692.

Robinson, D., 1994. The responses of plants to non-uniform supplies of nutrients. New Phytol. 127:635-674.

Rouached, H., M. Wirtz, R. Alary, R. Hell, A.B. Arpat, J.-C. Davidian, P. Fourcroy, and P. Berthomieu, 2008. Differential regulation of the expression of two high-affinity sulfate Transporters, SULTR1.1 and SULTR1.2, in Arabidopsis. Plant Physiol., 147:897-911.

Rouillon, A., Y. Surdin-Kerjan, and D. Thomas, 1999.Transport of sulfonium compounds. Characterization of the S-adenosylmethionine and Smethylmethionine permeases from the yeast Saccharomyces cerevisiae. J. Biol. Chem. 274, 28096-28105. doi: 10.1074/jbc.274.40.28096.

Sauter, M., B. Moffatt, M. C. Saechao, R. Hell, and M. Wirtz, 2013. Methionine salvage and Sadenosylmethionine: essential links between sulfur, ethylene and polyamine biosynthesis. Biochem. J. 451: 145-154. doi: 10.1042/BJ20121744.

Sawada, Y., A. Kuwahara, M. Nagano, T. Narisawa, A. Sakata, K. Saito, et al., 2009a. Omics-based approaches to methionine side chain elongation in Arabidopsis: characterization of the genes encoding methylthioalkylmalate isomerase and methylthioalkylmalate dehydrogenase. Plant Cell Physiol. 50: 1181-1190. doi: 10.1093/pcp/pcp079.

Sawada, Y., K.Toyooka, A. Kuwahara, A. Sakata, M. Nagano, K. Saito, et al., 2009b. Arabidopsis bile acid: sodium symporter family protein 5 is involved in methionine-derived glucosinolate biosynthesis. Plant Cell Physiol. 50: 1579-1586. doi: 10.1093/pcp/pcp110.

Schachtman, D.P., R. Shin, 2007. Nutrient sensing and signaling: NPKS. Annu Rev Plant Biol 58:4769

Schnaubelt, D., P. Schulz, M. A. Hannah, R. E. Yocgo, and C. H. Foyer, 2013. A phenomics approach to the analysis of the influence of glutathione on leaf area and abiotic stress tolerance in Arabidopsis thaliana. Front. Plant Sci. 4:416. doi: 10.3389/fpls.2013.00416.

Schulte, M., P. Von Ballmoos, H. Rennenberg, and C. Herschbach, 2002. Life-long growth of Quercus ilex L. at natural $\mathrm{CO} 2$ springs acclimates sulphur, Nitrogen and carbohydrate metabolism of the progeny to elevated pCO2.Plant, Cell Environ. 25: 1715-1727.

Schuster, J., T. Knill, M. Reichelt, J. Gershenzon, and S. Binder, 2006. BRANCHED-CHAIN AMINOTRANSFERASE4 is part of the chain elongation pathway in the biosynthesis of methionine-derived glucosinolates in Arabidopsis. Plant Cell 18: 2664-2679. doi: 10.1105/tpc.105.039339.

Shen, B., C. Li, and M. C. Tarczynski, 2002. High free-methionine and decreased lignin content result from a mutation in theArabidopsis S-adenosyl-L-methionine synthetase 3 gene. Plant J. 29: 371380. doi: 10.1046/j.1365-313X.2002.01221.x.

Shibagaki, N., and A. R. Grossman, 2004. Probing the function of STAS domains of the Arabidopsis sulfate transporters. J. Biol. Chem. 279: 30791-30799. doi: 10.1074/jbc.M403248200.

Shibagaki, N., and A. R. Grossman, 2006. The role of the STAS domain in the function and biogenesis of a sulfate transporter as probed by random mutagenesis. J. Biol. Chem. 281: 22964-22973. doi: 10.1074/jbc.M603462200. 
Shibagaki, N., and A. R. Grossman, 2010. Binding of cysteine synthase to the STAS domain of sulfate transporter and its regulatory consequences. J. Biol. Chem. 285: 25094-25102. doi: 10.1074/jbc.M110.126888.

Shibagaki, N., A. Rose, J. P. Mcdermott, T. Fujiwara, H. Hayashi, T. Yoneyama, et al., 2002. Selenateresistant mutants of Arabidopsis thaliana identify Sultr1;2, a sulfate transporter required for efficient transport of sulfate into roots. Plant J. 29: 475-486. doi: 10.1046/j.09607412.2001.01232.x.

Silke, C, W., and K. Stanislav, 2015. Are changes in sulfate assimilation pathway needed for evolution of C4photosynthesis.Plant Sc., 5: 1-8.

Smith, F. W., P. M. Ealing, M. J. Hawkesford, and D. T. Clarkson, 1995. Plant members of a family of sulfate transporters reveal functional subtypes. Proc. Natl. Acad. Sci. U.S.A. 92: 9373-9377. doi: 10.1073/pnas.92.20.9373

Smith, F. W., M. J. Hawkesford, P. M. Ealing, D. T. Clarkson, P. J. Vanden Berg, A. R. Belcher, et al., 1997. Regulation of expression of a cDNA from barley roots encoding a high affinity sulphate transporter. Plant J. 12: 875-884. doi: 10.1046/j.1365-313X.1997.12040875.x.

Smith, I. K., 1980. Regulation of sulfate assimilation in tobacco cells: effect of nitrogen and sulfur nutrition on sulfate permease and O-acetylserine sulfhydrylase. Plant Physiol. 66: 877-883. doi: 10.1104/pp.66.5.877.

Sønderby, I.E., Hansen, B.G., Bjarnholt, N., Ticconi,C.,Halkier,B.A.,and Kliebenstein, D.J., 2010. AsystemsbiologyapproachidentifiesaR2R3 MYB gene sub family with distinct and overlapping functions in regulation of aliphatic glucosinolates. PLoS ONE 2:e1322. doi:10.1371/journal.pone.0001322.

Song, W. Y., J. Park, D. G. Mendoza-Cozatl, M. Suter-Grotemeyer, D. Shim, S. Hortensteiner, et al., 2010. Arsenic tolerance in Arabidopsis is mediated by two ABCC-type phytochelatin transporters. Proc. Natl. Acad. Sci. U.S.A. 107: 21187-21192. doi: 10.1073/pnas.1013964107.

South, P. F., A. P. Cavanagh, H. W. Liu, and D. R. Ort, 2019. Synthetic glycolate metabolism pathways stimulate crop growth and productivity in the field. Science, 363, eaat9077 .

Stipanuk, M. H., 2004. Sulfur amino acid metabolism: pathways for production and removal of homocysteine and cysteine. Annu. Rev. Nutr. 24: 539-577.

Sunarpi, and J. W. Anderson, 1996. Effect of sulfur nutrition on the redistribution of sulfur in vegetative soybean plants, Plant Physiology, 112(2): 623-631.

Tabe, L. M., and M. Droux, 2001. Sulfur assimilation in developing lupin cotyledons could contribute significantly to the accumulation of organic sulfur reserves in the seed. Plant Physiol. 126, 176187. doi: 10.1104/pp.126.1.176.

Tada, Y., S.H. Spoel, K. Pajerowska-Mukhtar, Z. Mou, J. Song, C. Wang, J. Zuo, and X. Dong, 2008. Plant immunity requires conformational charges of NPR1 via S-nitrosylation and thioredoxins. Science 321:952-956.

Takahashi, S. and N. Murata, 2008. How do environmental stresses accelerate photo inhibition Trends in Plant Science, 13(4):178-182.

Takahashi, H., P. Buchner, N. Yoshimoto, M. J. Hawkesford, and S. H. Shiu, 2011a. Evolutionary relationships and functional diversity of plant sulfate transporters. Front. Plant Sci. 2:119. doi: 10.3389/fpls.2011.00119.

Takahashi, H., S. Kopriva, M. Giordano, K. Saito, and R. Hell, 2011. Sulfur assimilation in photosynthetic organisms: molecular functions and regulations of transporters and assimilatory enzymes. Annu. Rev. Plant Biol. 62: 157-184. doi: 10.1146/annurev-arplant-042110-103921.

Takahashi, H., S. Kopriva, M. Giordano, K. Saito, and R. Hell, 2011b. Sulfur assimilation in photosynthetic organisms: molecular functions and regulations of transporters and assimilatory enzymes. Annu. Rev. Plant Biol. 62:157-184. doi: 10.1146/annurev-arplant-042110-103921.

Takahashi, H., A. Watanabe-Takahashi, F. W. Smith, M. Blake-Kalff, M. J. Hawkesford, and K. Saito, 2000. The roles of three functional sulphate transporters involved in uptake and translocation of sulphate in Arabidopsis thaliana. Plant J. 23, 171-182. doi: 10.1046/j.1365-313x.2000.00768.x.

Takahashi, H., M. Yamazaki, N. Sasakura, A. Watanabe, T. Leustek, J. A. Engler, et al., 1997. Regulation of sulfur assimilation in higher plants: a sulfate transporter induced in sulfate-starved roots plays a central role in Arabidopsis thaliana. Proc. Natl. Acad. Sci. U.S.A. 94, 11102-11107. doi: 10.1073/pnas.94.20. 11102 . 
Tamara, G., and K. Stanislav, 2014. Transporters in plant Sulfur metabolism, Plant Sc., .5: 1-16 Doi $10.3389 /$ fpls.2014 00442 .

Tan, Q., L. Zhang, J. Grant, P. Cooper, and M. Tegeder, 2010. Increased phloem transport of Smethylmethionine positively affects sulfur and nitrogen metabolism and seed development in pea plants. Plant Physiol. 154: 1886-1896. doi: 10.1104/pp.110.166389.

Tandon, H.L.S., 1986. Sulfur Research and Agricultural Production in India. 2nd ed., Fertilizer Development and Consultation Organisation, New Delhi, p.76.

Tegeder, M., 2012. Transporters for amino acids in plant cells: some functions and many unknowns. Curr. Opin. Plant Biol. 15: 315-321. doi: 10.1016/j.pbi.2012.02.001.

Tejada-Jimenez, M., A. Llamas, E. Sanz-Luque, A. Galvan, and E. Fernandez, 2007. A high-affinity molybdate transporter in eukaryotes. Proc. Natl. Acad. Sci. U.S.A. 104: 20126-20130. doi: 10.1073/pnas.070 4646104.

Tomatsu, H., J. Takano, H. Takahashi, A. Watanabe-Takahashi, N. Shibagaki, and T. Fujiwara, 2007. An Arabidopsis thaliana high-affinity molybdate transporter required for efficient uptake of molybdate from soil. Proc. Natl. Acad. Sci. U.S.A. 104, 18807-18812. doi: 10.1073/pnas.0706373104.

Triantaphylid 'es, C., and M. Havaux, 2009. Singlet oxygen in plants: production, detoxification and signaling, Trends in Plant Science, 14(4): 219-228.

Vatamaniuk, O. K., S. Mari, Y. P. Lu, and P. A. Rea, 1999. AtPCS1, a phytochelatin synthase from Arabidopsis: isolation and in vitro reconstitution. Proc. Natl. Acad. Sci. U.S.A. 96, 7110-7115. doi: 10.1073/pnas.96.12.7110.

Vauclare, P., S. Kopriva, D. Fell, M. Suter, L. Sticher, P. von Ballmoos, U. Krahenbu“hl, R.O. den Camp, and C. Brunold, 2002. Flux control of sulphate assimilation in Arabidopsis thaliana: adenosine 50-phosphosulphate reductase is more susceptible than ATP sulphurylase to negative control by thiols. Plant J. 31:729-740.

Wachter, A., S. Wolf, H. Steininger, J. Bogs, and T. Rausch, 2005. Differential targeting of GSH1 and GSH2 is achieved by multiple transcription initiation: implications for the compartmentation of glutathione biosynthesis in the Brassicaceae. Plant J. 41:15-30. doi: 10.1111/j.1365-313X.2004. 02269.x.

Walker, C., and E.J. Boothe, 2003. Sulphur nutrition and oilseed quality. In: Abrol YP, Ahmad A (eds.Sulphur in plants. Kluwer Academic Publishers, Dordrecht, 323-340.

Wallsgrove, R. M., P. J. Lea, and B. J. Miflin, 1983. Intracellular localization of aspartate kinase and the enzymes of threonine and methionine biosynthesis in green leaves. Plant Physiol. 71, 780 784. doi: 10.1104/pp.71.4.780.

Watanabe, M., M. Kusano, A. Oikawa, A. Fukushima, M. Noji, and K. Saito, 2008. Physiological roles of the beta-substituted alanine synthase gene family in Arabidopsis. Plant Physiol. 146, 310-320. doi: 10.1104/pp.107. 106831.

Yang, Y., J.S. Yuan, J. Ross, J.P. Noel, E. Pichersky, and F. Chen, 2006. An Arabidopsis thaliana Methyltransferase capable of methylating farnesoic acid. Arch. Biochem. Biophys., 448:123132.

Yarmolinsky, D., G. Brychkova, A. Kurmanbayeva, A. Bekturova, Y. Ventura, I. Khozin-Goldberg, et al., 2014. Impairment in sulfite reductase leads to early leaf senescence in tomato plants. Plant Physiol. 165: 1505-1520. Doi: 10.1104/pp.114.241356.

Yoshimoto, N., E. Inoue, K. Saito, T. Yamaya, and H. Takahashi, 2003. Phloem-localizing sulfate transporter, Sultr1;3, mediates re-distribution of sulfur from source to sink organs in Arabidopsis. Plant Physiol., 131:1511-1517.

Yoshimoto, N., H. Takahashi, F.W. Smith, T. Yamaya, and K. Saito, 2002.Two distinct high-affinity sulfate transporters with different inducibilities mediate uptake of sulfate in Arabidopsis roots. Plant J. 29:465-473.

Yoshimoto, N., E. Inoue, A. Watanabe-Takahashi, K. Saito, and H. Takahashi, 2007. Posttranscriptional regulation of high-affinity sulfate transporters in Arabidopsis by sulfur nutrition. Plant Physiol. 145, 378-388. doi: 10.1104/pp.107.105742.

Zechmann, B., F. Mauch, L. Sticher, and M. Muller, 2008. Subcellular immunocytochemical analysis detects the highest concentrations of glutathione in mitochondria and not in plastids. J. Exp. Bot. 59: 4017-4027. doi: 10.1093/jxb/ern243. 
Zhang, B., R. Pasini, H. Dan, N. Joshi, Y. Zhao, T. Leustek, et al., 2014. Aberrant gene expression in the Arabidopsis SULTR1;2 mutants suggests a possible regulatory role for this sulfate transporter in response to sulfur nutrient status. Plant J. 77:185-197. doi: 10.1111/tpj.12376.

Zhang, M. Y., A. Bourbouloux, O. Cagnac, C. V. Srikanth, D. Rentsch, A. K. Bachhawat, et al., 2004. A novel family of transporters mediating the transport of glutathione derivatives in plants. Plant Physiol. 134: 482-491. doi: 10.1104/pp.103.030940.

Zhao, J., D. Huhman, G. Shadle, X.-Z. He, L. W. Sumner, Y. Tang, et al., 2011. MATE2 mediates vacuolar sequestration of flavonoid glycosides and glycoside malonates in Medicago truncatula. Plant Cell 23, 1536-1555. doi: 10.1105/tpc.110.080804

Zhou, W., L. Wei, J. Xu, H. Zuber, Davidian, J. C., Aubert, G., Aime, D., Belghazi, M., Lugan, R., et al., 2010. The seed composition of Arabidopsis mutants for the group 3 sulfate transporters indicates a role in sulfate translocation within developing seeds. Plant Physiol. 154: 913-926. doi: 10.1104/pp.110. 162123. 